\title{
ESTIMATING TRANSITION PROBABILITIES \\ FROM AGGREGATE SAMPLES AUGMENTED \\ BY HAPHAZARD RECAPTURES
}

\author{
D. L. Hawkins \\ C.-P. Han \\ J. Eisenfeld \\ Mathematics Department \\ University of Texas at Arlington
}

Technical Report \#302 


\title{
Estimating Transition Probabilities from Aggregate Samples Augmented by Haphazard Recaptures
}

\author{
D. L. Hawkins \\ C.- P. Han \\ J. Eisenfeld
}

Department of Mathematics

University of Texas at Arlington

Arlington, TX 76019 USA

Abstract

Transition probabilities provide a convenient summary of changes in a categorical trait over time in a population. The difficulties of estimating such probabilities based on only aggregate data from repeated sampling are well known. We give here a method for augmenting aggregate data with haphazard recapture data, which can dramatically improve the estimation precision of transition probabilities. The method requires a rather high sampling fraction to provide sufficient numbers of recaptures. It is based on a generalized nonlinear least squares strategy which yields transition probability estimates preserving their natural parameter space, and which are asymptotically efficient. The asymptotic theory is given under finite population sampling assumptions which are typical in practice.

1. Introduction

The problem of measuring change over time, based on repeated samples, in a population parameter descriptive of a trait $Z$ is an old one. It is widely recognized (see eg. Cochran (1977), Lee et al. (1977)) that a better estimate of the change in the parameter is obtained if one can measure $Z$ on the same units at each stage of sampling (so that change at the unit level is measured), than if one can only measure $Z$ in aggregate samples of units at each stage. However, logistical constraints often preclude tracking individual units through time, so that aggregate sampling for assessment of change is the best one can do.

For the case where $Z$ is a qualitative trait, we discuss here a strategy for estimating transition probabilities from aggregate data, augmented by haphazard recaptures (i.e. units which haphazardly occur in the samples at two consecutive stages of the sampling). Such augmentation 
is motivated by the generally poor estimates which result from using only the aggregate data. However, for such recaptures to be available in sufficient numbers to be useful, the sampling fraction must be reasonably high, so this strategy will not always be viable. We have applied it here (see Section 3 ) to a situation with sampling fraction .2 with good results.

The paper is organized as follows. The method is detailed in section 2. In 2.1 we use a finite population sampling ( $f p s$ ) framework for defining transition probabilities relative to a time-homogeneous Markov model. In 2.2 we develop an estimation scheme by incorporating the fps estimates of these parameters into a nonlinear regression model in order to efficiently estimate the transition probabilities while preserving their natural parameter space. We then obtain the limit distribution of the transition probability estimates as the population size $N \rightarrow \infty$. Unfortunately the theory of the asymptotic distributions of nonlinear least squares estimates in our non-standard setup was not available to the authors' knowledge, and had to be derived. This is done in 2.5. An example application is given in Section 3. Brief sketches of the proofs of the main theorems are given in the appendix. Complete proofs and other details are available in the companion technical report Hawkins et al. (1995) (henceforth called HHE).

\section{Methods}

\subsection{The Target Paraneters.}

We adopt a $f p s$ strategy. Let $l=1, \cdots, N$ index the members of the finite population of units under study. Let $t=1, \cdots, r$ index the sampling waves. Let $\mathrm{Z}$ be a qualitative trait with $m \geq 2$ categories defined for each unit. For $1 \leq i \leq m$, let $Z_{l t i}=1$ if unit $l$ has $Z=i$ at wave $t$, and otherwise be zero. Finally define the $m \times 1$ vector ${\underset{Z}{Z}}_{l t}^{0}=\left(Z_{l t 1}, \cdots, Z_{l t m}\right)^{T}$. (Note that $\underset{\sim}{Z_{l t}^{0}}$ is nonrandom.)

We introduce a time-homogeneous Markov model as follows. Define the $m \times 1$ vector parameter

$$
\underline{q}_{N t}^{0}=\frac{1}{N} \sum_{l=1}^{N} Z_{l t}^{0},
$$


whose typical element $\mathrm{q}_{N t i}^{0}, 1 \leq i \leq m$ is the fraction of the population with

$Z=i$ at wave $t$. Consider first a more general time-dependent Markov model consisting of the assertion that the sequence $\underline{q}_{N 1}^{0}, \cdots, \underline{q}_{N r}^{0}$ satisfies

$$
\underline{q}_{N, t+1}^{0}=\underline{P}_{t, t+1}^{T} \underline{q}_{N}^{0}, t=1 \cdots, r-1
$$

for some $m \times m$ transition matrices $P_{t, t+1}=\left(p_{i j}^{t, t+1}: i, j=1, \cdots, m\right)$. Model (2.1) is vacuous unless there actually exist such transition matrices $P_{t, t+1}$ defined in terms of relevant population quantities. To see that this is indeed the case, define

$$
p_{i j}^{t, t+1}=\frac{1}{N} \sum_{l=1}^{N} Z_{l t i} Z_{l, t+1, j} / \frac{1}{N} \sum_{l=1}^{N} z_{l t i}
$$

Then it may be verified that (2.1) holds with $p_{i j}^{t, t+1}$ defined by (2.2). Thus, $p_{i j}^{t, t+1}$ represents the fraction of the sub-population satisfying $Z=i$ at wave $t$, who subsequently satisfied $Z=j$ at wave $t+1$.

The time homogeneity of the Markov model (2.1) consists of $p_{i j}^{t, t+1}$ being the same for all t; i.e. $p_{i j}^{t, t+1}=: p_{i j}, t=1, \cdots, r-1$. If this condition holds, then, it may be verified (see HHE) that $p_{i j}$ must have the representation.

$$
p_{i j}=\sum_{t=1}^{r-1} \sum_{l=1}^{N} z_{l t i} Z_{l, t+1, j} / \sum_{t=1}^{r-1} \sum_{l=1}^{N} z_{l t i .}
$$

Since $p_{i j}=p_{i j}^{t, t+1}, t=1, \cdots, r-1$ must satisfy (2.1), time homogeneity implies that (2.1) holds with $\underline{P}=\left(p_{i j}\right)$ :

$$
\underline{q}_{N, t+1}^{0}=\underline{P}^{T} \underline{q}_{N t}^{0}, \quad t=1, \cdots, r-1
$$

The ensuing methods assume model (2.4) - i.e. the time homogeneous Markov model. The representation (2.3) is utilized in Section 2.4 to construct estimates of the $p_{i j}$.

A few comments about (2.4) are in order here.

Since we are using a finite population sampling approach, the $\underline{q}_{t}^{0}$ 's 
are not, strictly speaking, probability distributions, so the $p_{i j}$ 's are not, strictly speaking, transition probabilities. Rather, since the $\underline{q}_{t}^{0}$ 's are summaries of the distribution of the (non-random) trait $Z$ in the population at the waves $t, p_{i j}$ should be viewed as a summary measure of between-waves movement across the values of $Z$ at the population level. Of course, the $p_{i j}$ 's are particularly effective measures of change, since by their conditional nature they avoid problematic issues of unequal initial distributions $\underline{q}_{1}^{0}$ when comparing changes across several study populations. (2) The view of the $p_{i j}$ 's in comment (1) above is in contrast with that in other treatments of the aggregate data problem in the literature where (see e.g. Kabfleisch and Lawless (1984)) the sequence $\left\{Z_{l t i}: t=1, \cdots, r\right\}$ for unit $l$ is assumed to be stochastic, specifically a realization of a Markov chain governed by a transition matrix of probabilities $p_{i j}$ common to a conceptual population represented by unit $l$. The essential difference between views (1) and (2) is modeling at the population level (as in (1)) rather than at the unit level (as in (2)).

To avoid linear redundancies in our estimation maneuvers, we can restrict to "sufficient parts" of the parameters. To this end, define $\underline{q}_{t}$ as the $(m-1) \times 1$ vector containing the first $m-1$ elements of $\underline{q}_{t}^{0}$. Similarly, for $i=1, \cdots, m$ let $\underline{p}_{i}^{T}=\left(p_{i 1}, \cdots, p_{i, m-1}\right)$ (recalling that $\left.p_{i m}=1-\sum_{j=1}^{m-1} p_{i j}\right)$.

\subsection{The Estimation Scheme}

Standard approaches to the problem of estimating the $p_{i j}$ 's from aggregate data are based on (2.4) with estimates plugged in for the $q_{t}$ 's (see Lee et al (1977), Kabfleish \& Lawless (1984)). These methods either fail to constrain the $p_{i j}$ 's to their natural parameter space (Kabfleish \& Lawless (1984)) or fall short of offering statistical properties of resulting constrained estimates (Lee et al). In either case the estimates perform poorly - see Lawless \& McLeish (1984).

The approach here uses a reparameterization of the $p_{i j}$ 's to preserve their parameter space, yielding a nonlinear model in the new parameters $\beta_{i j}$ in place of (2.4). The model is then augmented (in the Goldberger - Theil 
sense of combining information) with the "recapture" estimates of the $p_{i j}$ 's, for which an asymptotic expectation model in terms of the $\beta_{i j}$ 's may be formulated. The augmented model parameters $\beta_{i j}$ are then efficiently estimated by generalized nonlinear least squares (gls), using an efficient weight matrix derived under our fps assumptions. Delta method approximations are used to produce the covariance matrix of the estimated $p_{i j}$ 's.

The remainder of this section defines the key features of the nonlinear model described above. Details of the estimates involved are given in section 2.4. In this direction let $\widehat{q}_{t}, t=1, \cdots, r$ denote the estimate of $\underline{q}_{t}$ and let $\underline{\hat{p}}_{i}$ denote the estimate of $\underline{p}_{i}, i=1, \cdots, m$.

The augmented reparameterized nonlinear model has the form

$$
\underline{Y}=\underline{F}\left(\underline{\beta} ; \underline{\widehat{q}}^{*}\right)+\underline{\epsilon} \text {, where }
$$

$\underline{Y}=\left[\begin{array}{c}\underline{Y}_{1} \\ \cdots \\ \underline{Y}_{2}\end{array}\right] \begin{aligned} & (r-1)(m-1) \times 1 \\ & m(m-1) \times 1\end{aligned}, \quad \underline{\epsilon}=\left[\begin{array}{c}\underline{\epsilon}_{1} \\ \cdots \\ \underline{\epsilon}_{2}\end{array}\right]$,

$\underline{F}=\left[\begin{array}{cc}\underline{F}_{1}\left(\underline{\beta} ; \underline{\hat{q}}^{*}\right) \\ \cdots \\ \underline{F}_{2}(\underline{\beta})\end{array}\right] \quad \begin{aligned} & (r-1)(m-1) \times 1 \\ & m(m-1) \times 1\end{aligned}$,

$\underline{\widehat{q}}^{*}=\left[\underline{\widehat{q}} \frac{T}{1}, \cdots, \underline{\widehat{q}} T\right]^{T} T \quad(r(m-1) \times 1)$,

$\underline{Y}_{1}=\underline{\sim}_{\widehat{q}^{*}}^{*}=\left[\begin{array}{c}\widehat{\underline{q}}_{2}^{*} \\ \cdots \\ \hat{\underline{q}}_{r}^{*}\end{array}\right], A=\left[\underline{0}_{(r-1)(m-1) \times(m-1)}: \underline{I}_{(r-1)(m-1)}\right]$

$\underline{Y}_{2}=\left[\hat{p}_{1}^{T}, \cdots, \underline{\hat{p}} \underset{m}{T}\right]^{T} \quad(m(m-1) \times 1)$

Thus model (2.5) has two parts, the first part representing (via $F_{1}$ ) the aggregate estimates in $\underline{Y}_{1}$ in terms of model (2.4), the second part representing (via the mean function $\underline{F}_{2}$ ) the transition probability estimates $\underline{Y}_{2}$ from the haphazard recaptures. 
The mean functions $F_{1}\left(\beta_{\sim} ; \widehat{\widehat{q}}^{*}\right)$ and $F_{2}(\underset{\sim}{\beta})$ involve the reparameterization of the $p_{i j}$ 's in terms of $\underline{\beta}=\left(\beta_{11}, \cdots, \beta_{1, m-1}, \beta_{21}, \cdots, \beta_{2, m-1}, \cdots, \beta_{m 1}, \cdots, \beta_{m, m-1}\right)^{T}$ $(m(m-1) \times 1)$ as follows:

$p_{i j}=p_{i j}(\beta)=e^{\beta i j} /\left[1+\sum_{k=1}^{m-1} e^{\beta i k}\right], \quad 1 \leq j \leq m-1, \quad 1 \leq i \leq m$.

Clearly $0 \leq p_{i j}(\underline{\beta}) \leq 1, \sum_{j=1}^{m-1} p_{i j}(\underline{\beta}) \leq 1$ for each $i$ and

$p_{i m}(\underline{\beta})=1 /\left[1+\sum_{k=1}^{m-1} e^{\beta i k}\right]$.

Also, $\underset{\beta}{\beta} \in I R^{m(m-1)}$ is an unconstrained parameter.

To get the elements of $\underline{F}_{1}\left(\underline{\beta} ; \underline{\widehat{q}}^{*}\right)$, write

$$
\underline{F}_{1}\left(\underline{\beta} ; \underline{\underline{q}}^{*}\right)=\left[\begin{array}{c}
F_{11} \\
F_{12} \\
\cdots \\
\underline{F}_{1, r-1}
\end{array}\right] \quad, \underline{F}_{1 t}=\left[\begin{array}{c}
F_{1 t 1} \\
F_{1 t 2} \\
\cdots \\
F_{1 t, m-1}
\end{array}\right]
$$

and then write out the right side of (2.4) in scalar form to get

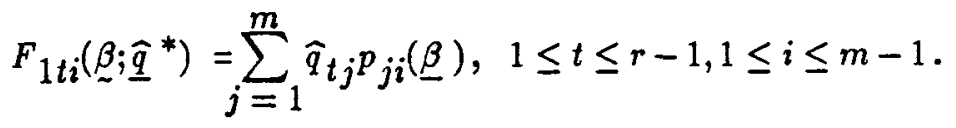

This part (from aggregate data) of model (2.5) arises essentially by plugging estimates of the $\underline{q}_{t}^{\prime} s$ into both sides of (2.4). The justification for casting this part of the model in the form (2.5) is that the difference $\underline{\epsilon}_{-1}=\underline{Y}_{1}-\underline{F}_{1}\left(\underline{\beta} T ; \underline{\widehat{q}}^{*}\right)$ may be considered as being approximately multivariate normal with zero mean if model (2.4) actually holds, based on $f p s$ central limit theory (see Lemma 2.3 in Section 2.5.3).

The "recaptures" part of (2.5) $\left(\underline{Y}_{2}\right.$ and $\left.\underline{F}_{2}(\beta)\right)$ is justified as follows. The estimate $\underline{\hat{p}}_{i}$ of $\underline{p}_{i}$ may be considered to be approximately multivariate normal with mean $\underline{p}_{i}$ and variance matrix $\operatorname{Var}\left(\underline{\widehat{p}}_{i}\right)$ by virtue of $f p s$ central 
limit theory (see Theorem 2.1 of Section 2.5.2). Thus, if we define $\underline{F}_{2}(\beta)=\left[\begin{array}{c}F_{21} \\ F_{22} \\ \vdots \\ F_{2 m}\end{array}\right], \quad \underline{F}_{2 k}=\left[\begin{array}{c}F_{2 k 1} \\ \vdots \\ F_{2 k, m-1}\end{array}\right]$, and

$F_{2 k j}(\underline{\beta})=p_{k j}(\underline{\beta}), 1 \leq k \leq m, 1 \leq j \leq m-1$

with $p_{k j}(\underline{\beta})$ given by $(2.10)$, then $\underline{F}_{2}(\beta)$ is the asymptotic mean of $\underline{Y}_{2}$ so long as (2.4) holds. The fact that $\underline{\epsilon}_{1}$ and $\underline{\epsilon}_{2}=\underline{Y}_{2}-\underline{\sim}_{2}(\underline{\beta})$ are approximately jointly normal supports suitably weighted least squares as an estimation strategy. However, compensation for the "random regressor" $\underline{\widehat{q}}^{*}$ in $\underline{\sim}_{1}$ will need to be made if an asymptotically optimal generalized least squares estimator $\widehat{\widehat{\beta}}_{G L S}$ is to be constructed; see section 2.5.3.

\subsection{The Sampling Design}

Since this directly impacts the form of the matrix $\operatorname{Var}(\underline{Y})$, we detail our assumptions here.

Briefly, we assume that at wave $t$, we select a simple random sample of size $n_{t}$, where $n_{t}$ is determined in advance. Further we assume that the samples at waves $t$ and $t^{\prime}$ are independent if $t \neq t^{\prime}$. Let $f_{t}=n_{t} / N$ denote the sampling fraction.

In more precise terms, for wave $t$ define random indicator variables $I_{l t}=1$ if unit $l$ is selected in the sample and $I_{l t}=0$ otherwise, $l=1, \cdots, N$. Let $\alpha_{l t}=E\left(I_{l t}\right)$ be the design probability. Then we assume that $\sum_{l=1}^{N} I_{l t}=n_{t}$, that $I_{l t}$ and $I_{l t}^{\prime} t^{\prime}$ are independent for $t \neq t^{\prime}$ and all $l, l^{\prime}$ and that $\alpha_{l t}=n_{t / N^{*}}$.

These assumptions are reasonable in our application in section 3 , but in principle the methods discussed here could be adapted to other sampling designs (e.g. unequal $\alpha_{l t}$ 's, correlated sampling indicators across waves, 
etc.). For brevity such extensions are not considered here.

\subsection{The Estimates of $q_{t}$ and $p_{i j}$}

Let

$$
\widehat{\widehat{q}}_{t}=\frac{1}{n_{t}} \sum_{l=1}^{N} Z_{l t} I_{l t}
$$

where $\underline{Z}_{l t}^{T}=\left(Z_{l t 1}, \cdots, Z_{l t, m-1}\right)$. Then under our assumptions in section 2.3 about the sampling process, $\underline{\underline{q}}_{t}$ is (design) unbiased for $\underline{q}_{t}$, with covariance matrix

$$
\operatorname{Var}\left(\underline{\underline{q}}_{t}\right)=\frac{1}{n_{t}}\left(1-f_{t}\right) \frac{1}{N-1} \sum_{l=1}^{N}\left(\underline{Z}_{l t}-\underline{q}_{t}\right)\left(\underline{Z}_{l t}-\underline{q}_{t}\right)^{T}
$$

A consistent estimate of $\operatorname{Var}\left(\hat{\underline{q}}_{t}\right)$ is

$$
\begin{aligned}
\widehat{V} \operatorname{ar}\left(\underline{\underline{q}}_{t}\right)= & \frac{1}{n_{t}}\left(1-f_{t}\right) \frac{1}{n_{t}-1} \sum_{l=1}^{N}\left(\underline{Z}_{l t}-\underline{\underline{q}}_{t}\right)\left(\underline{Z}_{l t}-\underline{\hat{q}}_{t}\right)^{T} I_{l t} \\
& =\frac{1}{n_{t}-1}\left(1-f_{t}\right)\left\{\operatorname{Diag}\left(\underline{\widehat{q}}_{t}\right)-\underline{\widehat{q}}_{t} \widehat{\widehat{q}}_{t}^{T}\right\},
\end{aligned}
$$

which requires that the sampling fractions $f_{t}$ be either known or ignorable.

By virtue of the independence of the $I_{l t}$ 's for distinct $t, \underline{q}_{t}$ and $\underline{\hat{q}}_{t}$ are independent if $t \neq t^{\prime}$, so $\operatorname{Var}\left(\underline{\widehat{q}}^{*}\right)=\operatorname{Diag}\left(\operatorname{Var}\left(\underline{\hat{q}}_{t}\right): t=1, \cdots, r\right)$ and $\operatorname{Var} \quad\left(\underline{Y}_{1}\right)=\underline{A}$ $\operatorname{Var}\left(\underline{\widehat{q}}^{*}\right) A^{T}$.

In view of (2.3), a natural estimate of $p_{i j}$ is $\widehat{p}_{i j}=R_{i j} / D_{i}$, where

$$
\begin{gathered}
R_{i j}=\sum_{t=1}^{r-1}\left(n_{t} n_{t+1}\right)^{-1} \sum_{l=1}^{N} z_{l t i} Z_{l, t+1, j} I_{l t} I_{l, t+1} \quad \text { and } \\
D_{i}=\sum_{t=1}^{r-1}\left(n_{t} n_{t+1}\right)^{-1} \sum_{l=1}^{N} z_{l t i} I_{l t} I_{l, t+1} .
\end{gathered}
$$

It is easy to check that $N \cdot R_{i j}$ and $N \cdot D_{i}$ are, respectively, unbiased for the numerator and denominator of $p_{i j}$ in (2.3). (This is the reason for using the divisor $n_{t} n_{t+1} / N$, whch is the expected number of recaptures, rather than the observed number $n_{t, t+1}$ of recaptures, which is a random variable.)

Define $\underline{\hat{p}}_{i}=:\left(\widehat{p}_{i 1, \ldots, \hat{p}_{i, m-1}}\right)^{T}$, which naturally estimates $\underline{p}_{i}$. The $\underline{\hat{p}}_{i}, i=1, \cdots, m$ will be arranged in the $\underline{Y}_{2}$ - component of the data vector in 
model (2.5) as shown in (2.9). Estimates of $\operatorname{Var}\left(\underline{Y}_{2}\right)$ and cov $\left(\underline{Y}_{1}, \underline{Y}_{2}\right)$ are developed in section 2.5.4.

Since the data will typically be available in some summarized form ( and not in terms of the indicators $\left.Z_{(t i}\right)$, we now give the sample estimates $\widehat{q}_{t i}$ and $\hat{p}_{i j}$ in terms of summarized data quantities.

The marginal aggregate data (for the $\underline{\underline{q}}_{t}$ 's) may be arranged into the form of Table 2.1.

Table 2.1. Marginal Aggregate Data Frequency Table

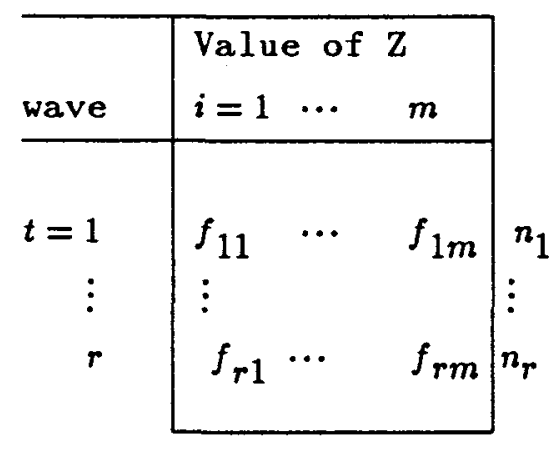

$$
\begin{aligned}
n_{t} & =\sum_{i=1}^{m} f_{t i} \\
f_{t i} & =\sum_{l=1}^{N} Z_{l t i} I_{l t}=\text { number of sampled units }
\end{aligned}
$$

with $Z=i$ at wave $t$

Of course, for $1 \leq t \leq r, \underline{\hat{q}}_{t}^{0}=\left[\widehat{q}_{t 1}, \cdots, \widehat{q}_{t m}\right]^{T}$, with $\widehat{q}_{t i}=f_{t i} / n_{t}$.

The recapture data may be arranged into the form of Table 2.2. 
Table 2.2 Transition Data from Haphazard Recaptures

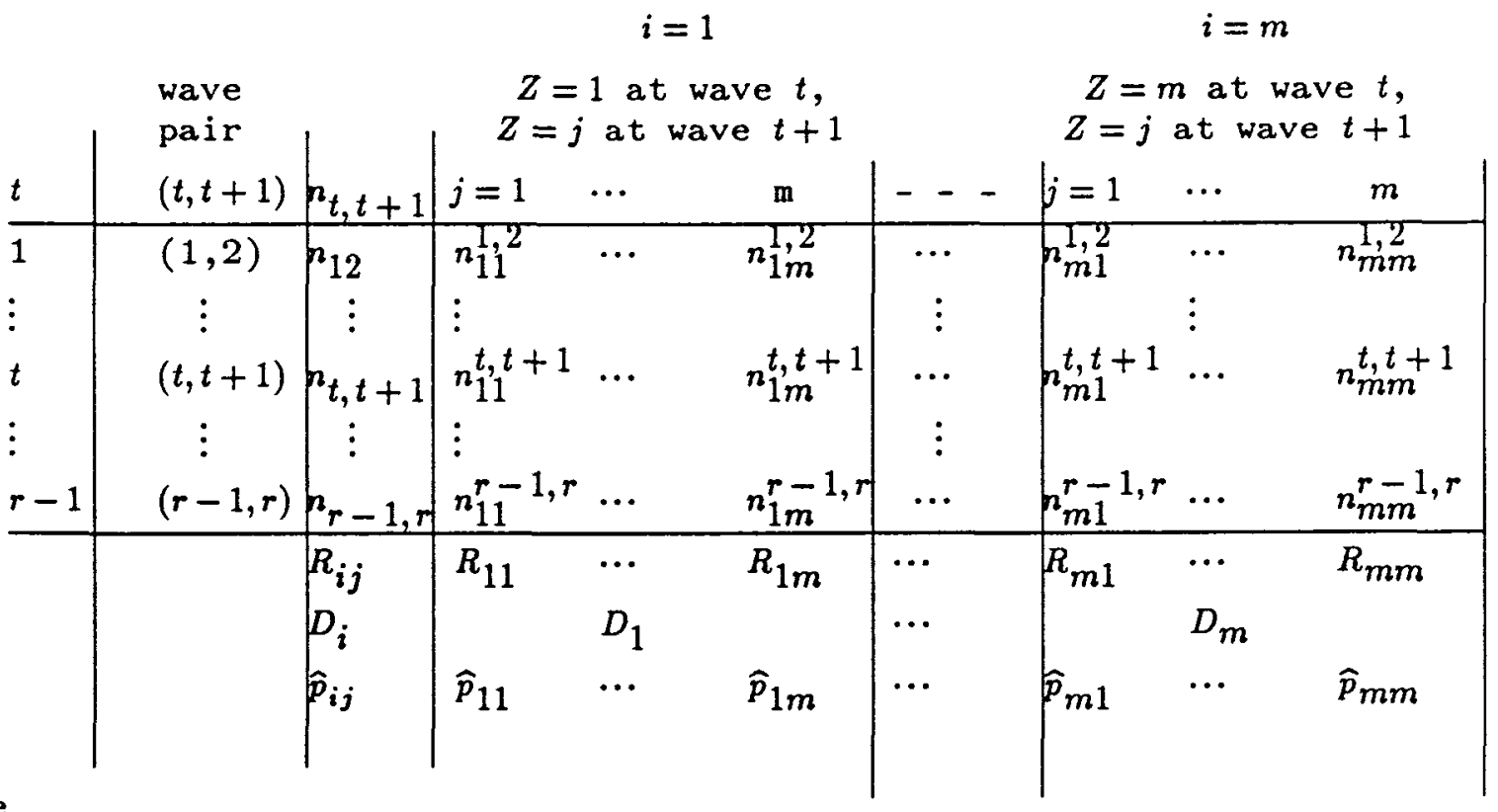

Here

$$
n_{i j}^{t, t+1}=\sum_{l=1}^{N} z_{l t i} Z_{l, t+1, j} I_{l t} I_{l, t+1}=\text { number of recaptures for wave pair }
$$

$(t, t+1)$ which had $Z=i$ at wave $t$ and $Z=j$ at wave $t+1$, and

$$
n_{t, t+1}=\sum_{i=1}^{m} \sum_{j=1}^{m} n_{i j}^{t, t+1} \text { is the total number of recaptures in the data for }
$$

wave pair $(t, t+1)$. The $R_{i j}$ 's are the weighted column sums

$R_{i j}=\sum_{t=1}^{r}\left(n_{t} n_{t+1}\right)^{-1} n_{i j}^{t, t+1}$, and $\hat{p}_{i j}=R_{i j} / D_{i}$. The quantity $D_{i}$ may be shown to equal $\sum_{j=1}^{m} R_{i j}$ 


\subsection{The Asymptotically Optimal Nonlinear Least Squares Estimate of $\underline{B}$ and}

its Asymptotic Distribution

\subsubsection{The Asymptotic Framework}

To obtain an asymptotic theory for the finite population sampling model, we must make assumptions about the behavior of certain population quantities as the population size $N \rightarrow \infty$. These assumptions are to some degree artificial, but serve as rough guides to the data requirements needed for reliable results.

We assume the following of the finite population trait vectors $Z_{l t}^{0} \equiv Z_{N l t}^{0}$ as $N \rightarrow \infty$ :

(A1) $\bar{Z}_{N t}^{0}=: \frac{1}{N} \sum_{l=1}^{N} Z_{N l t}^{0} \rightarrow \bar{Z}_{t}^{0}$, a finite vector, $t=1, \cdots, r$.

(A2) $\frac{1}{N} \sum_{l=1}^{N}\left({\underset{Z}{N l t}}_{N}^{0}-\bar{\sim}_{N t}^{0}\right)\left(Z_{N l t}^{0}-\bar{\sim}_{N t}^{0}\right)^{T} \rightarrow{\underset{\sim}{T}}_{t}^{0}$, a finite matrix, $t=1, \cdots, r$.

(A3) The wave sample sizes $n_{t}, 1 \leq t \leq r$, satisfy $n_{t}=n_{N} \cdot \lambda_{t}$, where $\lambda_{t}>0$, $\lambda_{1}+\cdots+\lambda_{r}=1$ and $n_{N \rightarrow \infty}$, but $n_{N} / N \rightarrow 0$, as $N \rightarrow \infty$. (Note that this implies $\left.\sum_{t=1}^{r} n_{t}=n_{N}\right)$

(A4) $n_{N}^{3 / 2} / N \rightarrow \infty$.

(A5) The marginal wave vectors $\left\{\underline{q}_{1}, \cdots, \underline{q}_{r-1}\right\} \operatorname{span} \mathbb{R}^{m}$ (so $r-1 \geq m$ must hold).

For later use let $g_{N t}=: n_{t} n_{t+1} / N$ and $g_{N}=\sum_{t=1}^{r} g_{N t}$.

In regard to these assumptions, (A4) implies (actually more than) that the expected number of repeats between waves $t$ and $t+1$, which is $n_{t} n_{t+1} / N$, is $O\left(n_{N}^{2} / N\right) \rightarrow \infty$ as $N \rightarrow \infty$. This is a necessary assumption if the $\widehat{\widehat{p}}_{i}$ 's are to be approximately normal. Assumption (A5), which is needed for both the consistency and asymptotic normality of the nonlinear least squares estimator, is discussed (essentially) in Kabfleisch and Lawless (1984). It implies that if the population is near a stationary distribution, then the methods discussed here will not work. 


\subsubsection{Asymptotic Normality of the Basic Estimates}

Since the result is used in the sequel, and is of interest in its own right, we first obtain the asymptotic joint normality of the components of $\underline{Y}$ in model (2.5). Of some note here are the different rates of convergence of the $\underline{Y}_{1}$ and $\underline{Y}_{2}$ components.

Put $\underline{W}_{N}^{T}=\left[\underline{\widehat{q}}_{N}^{* T}, \underline{Y}_{2 N}^{T}\right]$. (Note the addition of the subscript $N$ for these asymptotic results.) It will be convenient to write the $i^{t h}$ component $(1 \leq i \leq m) \underline{\hat{p}}_{i}$ of $\underline{Y}_{2 N}$ in the form $\underline{\hat{p}}_{i}=\underline{R}_{i} / D_{i}$, where

$$
\begin{gathered}
\underline{R}_{i}=\sum_{t=1}^{r-1} g_{N t}^{-1} \sum_{l=1}^{N} Z_{l t i} \underline{Z}_{l, t+1} I_{l t} I_{l, t+1}, \\
D_{i}=\sum_{t=1}^{r-1} g_{N t}^{-1} \sum_{l=1}^{N} Z_{l t i} I_{l t} I_{l, t+1} .
\end{gathered}
$$

Let

$$
\begin{gathered}
\underline{B}_{N}=\text { Blockdiag }\{n_{1}^{\frac{1}{2}} \underline{I}_{m-1}, \cdots, n_{r}^{\frac{1}{2}}, \underline{I}_{m-1}, \underbrace{\left.g^{\frac{1}{2}} \underline{I}_{m-1}, \cdots, g_{N}^{\frac{1}{2}} \underline{I}_{m-1}\right\}}_{N}, \\
\underline{R}_{i}^{*}=\lim _{N \rightarrow \infty} E\left(\frac{1}{N} \underline{R}_{i}\right), D_{i}^{*}=\lim _{N \rightarrow \infty} E\left(\frac{1}{N} D_{i}\right), \underline{q}^{*} \lim _{N \rightarrow \infty} E\left(\underline{\hat{q}}_{N}^{*}\right)
\end{gathered}
$$

(all assumed to exist), and set $\underline{p}_{i}^{*}=\underline{R}_{i}^{*} / D_{i}^{*}$.

Let $\underline{W}^{*}=\left[\underline{q}^{* T}, \underline{p}_{1}^{* T}, \cdots, \underline{p}_{m}^{* T}\right]^{T}$

Theoren 2.1. Under model (2.4) and assumptions (A1)-(A4),

$$
\underline{B}_{N}\left\{\underline{W}_{N}-\underline{W}^{*}\right\} \stackrel{\mathscr{L}}{\rightarrow} N\left(\underline{0}, \underline{\Gamma}_{W}\right) \text { as } N \rightarrow \infty
$$

for some p.d. covariance matrix $\underline{\Gamma}_{W}$.

The exact form of $\underline{\Gamma}_{W}$ is of no interest, since we shall never use it. The scaling matrix $\underline{B}_{N}$ gives the different rates of convergence of the aggregate estimate $\underline{\underline{q}}_{N}^{*}$ and the transition estimates $\underline{\hat{p}}_{i}$. 


\subsubsection{The Estimate of $\beta$ and its Asymptotic Distribution}

There are several complicating factors. First, the asymptotically optimal weight matrix (for $g l s$ ) depends on the random regressor $\underline{q}_{N}^{*}$ in model (2.5). Second, the sampling process assumed here ( $f p s$ ) is not the usual one (iid sampling) assumed for nonlinear least squares distribution theory, so the arguments needed to get the asymptotic distribution of the nonlinear least squares estimate are non-standard. Finally, the "data" vector $\underline{Y}$ in (2.5) contains two types of estimates: aggregate and transition estimates, which converge at different rates as $N \rightarrow \infty$.

The following general theorem accomodates these issues, and provides the framework by which we will establish the limit distribution of the gls estimator. The theorem generalizes a corresponding result in Kabfleisch and Lawless (1984) for the linear case and common convergence rates.

Theorem 2.2 Consider the model

$$
\underline{\underline{Y}}_{n}=\underset{\sim}{f}\left(\underline{\tilde{X}}_{n} ; \underline{\theta}_{T}\right)+\underline{\tilde{\varepsilon}}_{n}
$$

where $\tilde{\sim}_{n}$ is $s \times 1,{\underset{\sim}{X}}_{n}$ is $s \times a, \underline{\sim}_{T}$ is $v \times 1, \tilde{\epsilon}_{n}$ is $s \times 1$, and $\tilde{X}_{n}, \tilde{Y}_{n}$ and $\tilde{\epsilon}_{n}$ are random matrices satisfying (as $n \rightarrow \infty$ )

(i) $\tilde{X}_{n} \stackrel{p}{\rightarrow} \underset{\sim}{\tilde{H}}, \underline{\tilde{Y}}_{n} \stackrel{P}{\rightarrow} \underline{\tilde{G}}$, where $\underline{\tilde{H}}$, $\underline{\tilde{G}}$ are constant and satisfy $\underline{\tilde{G}}=\underset{\sim}{f}\left(\underset{\tilde{H}}{\tilde{\theta}} \underline{\theta}_{T}\right)$;

(ii) ${\underset{\sim}{B}}_{n} \tilde{E}_{n} \stackrel{\mathscr{L}}{\rightarrow} N_{s}\left(\underline{0}, \sum_{\epsilon}\right)$, where $\sum_{\epsilon}(s \times s)$ is positive definite $(p . d$.$) and {\underset{\sim}{*}}_{n}$ is an $s \times s$ diagonal matrix of scaling sequences (not necessarily all the same) such that there exists a real sequence $h_{n}>0, h_{n} \rightarrow 0$ for which $h_{n} \tilde{B}_{n} \rightarrow \tilde{B}$;

(iii) $\operatorname{rank}\left(\underset{\sim}{\tilde{B}} \underset{\sim}{f}\left(\tilde{H} ; \underline{\theta}_{T}\right)\right)=v$, where

$\underline{f}(\tilde{H} ; \underline{\theta})$ is the $s \times v \quad \underline{\theta}-$ Jacobian matrix of $\underset{\sim}{f}$ (assumed suitably differentiable).

Further, for arbitrary $s \times s$ p.d. $Q$, define 


$$
S_{Q}(\underline{\theta})=\left[\underline{\tilde{Y}}_{n}-\underset{\sim}{f}\left(\tilde{\tilde{X}}_{n} ; \theta\right)\right]^{T} \underline{\tilde{B}}_{n}^{T} \underline{\underline{\tilde{B}}} \underline{\underline{Y}}_{n}\left[\underline{\tilde{Y}}_{n}-\underline{f}\left(\underline{\tilde{X}}_{n} ; \underline{\theta}\right)\right]
$$

and let $\widehat{\theta}_{n}$ satisfy conditions (iv) and (v) below:

(iv) $\frac{d}{d \underline{\theta}} S_{q}\left(\hat{\theta}_{n}\right)=\underline{0}$, or equivalently,

$$
\dot{\leftrightarrow}^{T}\left(\underline{\tilde{X}}_{n} ; \underline{\hat{\theta}}_{n}\right) \underline{\tilde{B}}_{n}^{T} \underline{\underline{Q}} \underline{\tilde{B}}_{n}\left[\underline{\tilde{Y}}_{n}-\underline{f}\left(\underline{\tilde{X}}_{n} ; \underline{\hat{\theta}}\right)\right]=\underline{0} ;
$$

(v) ${\underset{\theta}{\theta}}_{n} \stackrel{P}{\rightarrow} \underset{\sim}{\theta} T$ as $n \rightarrow \infty$.

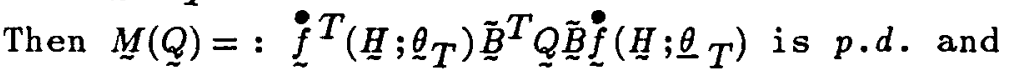

$h_{n}^{-1}\left(\underline{\hat{\theta}}_{n}-\underline{\sim}_{T}\right)^{\stackrel{\mathcal{L}}{\rightarrow}} N_{v}\left(\underline{0}, \underset{\sim}{\Gamma}\left(\underline{\theta}_{T} ; Q\right)\right)$ as $n \rightarrow \infty$,

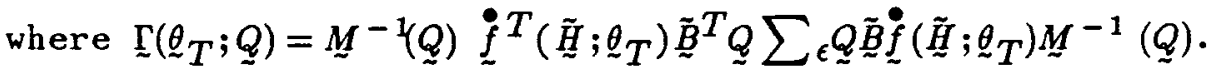

Further, if $Q=\sum \epsilon^{-1}$, then $\hat{\theta}_{n}$ is optimal (in the sense that $\Gamma\left(\theta_{T} ; \underline{Q}\right)-\Gamma\left(\underline{\theta}_{T} ; \sum_{\tilde{\epsilon}}^{-1}\right)$ is positive semi-definite for all p.d. $\left.Q\right)$ and $\Gamma\left(\theta_{T} ; \sum_{\epsilon}^{-1}\right)=M^{-1}\left(\sum_{\tau} \epsilon^{-1}\right)$. The asymptotic optimality continues to hold if $\underline{Q}=\sum_{\tilde{\epsilon}}^{-1}$, a consistent estimate of $\sum \epsilon^{-1}$.

To apply this result in the present setting let $s=\operatorname{dim}(Y)=(r+m-1)(m-1), \nu=m(m-1)$ and let $\underline{Q}(s \times s)$ denote an arbitrary p.d. matrix. The corresponding gls estimate $\underline{\hat{\beta}} Q$ of $\underset{\sim}{\beta}$ in (2.5) satisfies $\underline{\hat{\beta}}_{Q}=\underset{\beta}{\operatorname{argmin}} S_{N}(\underline{\beta})$, where, with

$$
\begin{aligned}
& \tilde{\sim}_{N}=B \operatorname{lockdiag}\{\underbrace{n_{N}^{\frac{1}{2}} I_{m-1}, \cdots, n_{N}^{\frac{1}{2}} \underline{I}_{m-1}}, \underbrace{g_{N}^{\frac{1}{2}} \underline{I}_{m-1}, \cdots, g_{N}^{\frac{1}{2}} I_{m-1}}\}, \\
& r-1 \text { blocks } \quad m \text { blocks } \\
& S_{N}(\underline{\beta})=: n_{N}^{-1}\left\{\underline{Y}_{N}-\underline{F}\left(\underline{\beta} ; \underline{\widehat{q}}_{N}^{*}\right)\right\}^{T} \underline{\tilde{B}}_{N}^{T} \underline{T} \underline{\tilde{B}}_{N}\left\{\underline{Y}_{N}-\underline{F}\left(\underline{\beta} ; \underline{\widehat{q}}_{N}^{*}\right)\right\}
\end{aligned}
$$

is the error sum of squares function. We shall apply Theorem 2.2 with

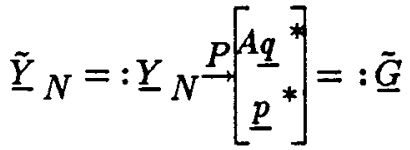

$$
\begin{aligned}
& \tilde{\tilde{X}}_{N}=: \underline{\widehat{q}}_{N}^{*} \stackrel{P}{\rightarrow} \underline{q}^{*}=: \underline{\tilde{H}} \text {, } \\
& \underline{\sim}_{\sim}^{f}\left(\tilde{X}_{N} ; \underline{\theta}\right)=: F\left(\underline{\beta} ; \underline{\widehat{q}}_{N}^{*}\right), \underline{\theta} \equiv \underline{\beta}, \underline{\theta}_{T} \equiv \underline{\beta}_{T}, v=\operatorname{dim}(\underline{\beta}),
\end{aligned}
$$


$\underline{\tilde{\epsilon}}_{N}=: \underline{\epsilon}_{N}=\underline{Y}_{N}-\underline{F}\left(\underline{\beta} T ; \underline{\widehat{q}}_{N}^{*}\right)$

$\tilde{B}=B$ lockdiag $\{\underbrace{\underline{I}_{m-1}, \cdots, I_{m-1}}_{r-1 \text { blocks }}, \underbrace{0 \cdot \underline{I}_{m-1}, \cdots, 0 \cdot \underline{I}_{m-1}}_{m \text { blocks }}\}$,

and $h_{N}=n_{N}-1 / 2$. (Here the convergences in (2.20) and (2.21) hold, under (2.4), as consequences of Theorem 2.1.) In Lemma 2.3 below it is shown that these quantities (as defined in the present setting) satisfy the conditions (ii) and (iii) of Theorem 2.2. Thus, for any fixed p.d. weight matrix $\underline{Q}$, any consistent minimizer of $S_{N}(\underline{\beta})$ in (2.19) (which via differentiability will necessarily satisfy its condition (iv)) will be asymptotically normal per Theorem 2.2. The consistency of $\underline{\hat{\beta}} Q$ is also established in Lemma 2.3.

Lemma 2.3 Under conditions (A1) - (A5) and model (2.4),

(a) $\tilde{B}_{N} \tilde{\tilde{\epsilon}}_{N} \stackrel{\mathscr{L}}{\rightarrow} N_{s}\left(\underline{0}, \sum_{\epsilon}\right)$ as $N \rightarrow \infty$, where

$\sum_{\epsilon}=\dot{\xi}\left(\underline{W}^{*}\right) \underset{\sim}{L} \Gamma_{W} L_{\sim}^{T} \dot{\xi}^{T}\left(\underline{W}^{*}\right)$,

$L=\underset{\sim}{D} \otimes \underline{\sim}_{m-1}, \underset{\sim}{D}=\operatorname{Diag}\left(\lambda_{1}{ }^{-\frac{1}{2}}, \cdots, \lambda_{r}{ }^{-\frac{1}{2}}, \underline{1}_{m}^{T}\right)$,

$\Gamma_{W}$ and ${\underset{W}{*}}^{*}$ are as in Theorem 2.1 , and

$\dot{\xi}\left(\underline{W}^{*}\right)=\left[\begin{array}{cc}A-\frac{\partial}{\partial \widehat{q}_{N}^{*}} F_{1}\left(\beta_{T} ; q^{*}\right) & 0 \\ \hdashline 0 & \underline{I}_{m(m-1)}\end{array}\right]$.

(b) $\operatorname{rank}\left\{\underset{\sim}{\tilde{B}} \underset{\underset{\sim}{\partial}}{\frac{\partial F}{\partial \tilde{\beta}}}\left(\beta_{T} ; q^{*}\right)\right\}=\nu=m(m-1)$.

(c) $\underline{\widehat{\beta}}_{Q N} \stackrel{p}{\rightarrow} \underline{\beta}_{T}$ as $N \rightarrow \infty$.

Thus by Theorem 2.2 we have the main result of this section. 
Theorem 2.4 Under conditions (A1)-(A5) and model (2.4), for any p.d. weight matrix $\underline{Q}, \underline{\widehat{\beta}} Q N$ minimizing (2.19) satisfies

$n{ }_{N}^{1 / 2}(\underline{\hat{\beta}} Q N-\underline{\beta} T) \stackrel{\mathcal{L}}{\rightarrow} N\left(\underline{0}, \Gamma_{\beta}(\underline{\beta} T ; \underline{Q})\right)$,

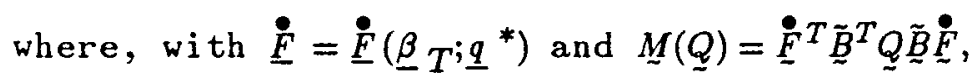

$\underline{\Gamma}_{\beta}\left(\underline{\beta}_{T} ; \underline{Q}\right)=\underline{M}^{-1}(\underline{Q}) \stackrel{\bullet}{F}^{T} \underline{\tilde{B}}^{T} \underline{Q} \sum \epsilon \underline{Q} \underset{\tilde{B}}{\stackrel{\bullet}{F}} \underline{M}^{-1}(\underline{Q})$

Corollary $\underline{2.5}$ With $\underline{Q}=\sum \epsilon^{-1}$ (or a consistent estimate of it), the asymptotically optimal gls estimate $\underline{\widehat{\beta}}_{N}$, defined as $\underline{\hat{\beta}} Q N$ with this choice of $\underline{Q}$, satisfies

$n_{N}^{1 / 2}\left(\underline{\widehat{\beta}}_{N}-\underline{\beta}_{T}\right)^{\stackrel{\ell}{\rightarrow}} N\left(0, \Gamma_{\beta}\left(\underline{\beta} T ; \sum \epsilon^{-1}\right)\right)$, where

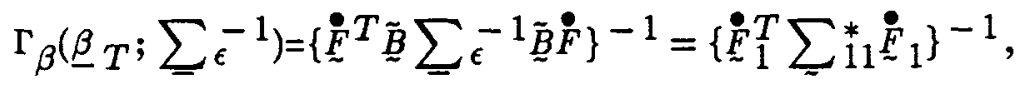

$\sum_{\tau} \epsilon^{-1}=\left[\begin{array}{cc}\sum_{11}^{*} & \sum_{12}^{*} \\ \sum_{12}^{*} T & \sum_{22}^{*}\end{array}\right], \quad \sum_{11}^{*}$ is $v \times v$ and $\stackrel{\bullet}{F}^{T}=\left[\stackrel{\bullet}{F}_{1}^{T}, \underset{\sim}{\stackrel{*}{T}}\right]$

Corollary 2.6. With $\underline{Q}=\underline{I}$, the ordinary least squares (ols) estimator $\underline{\hat{\beta}} I N$ satisfies

$n_{N}^{1 / 2}(\underline{\widehat{\beta}} I N-\underline{\beta} T) \stackrel{\mathscr{L}}{\rightarrow} N\left(\underline{0}, \Gamma_{\beta}(\underline{\beta} T ; \underline{I})\right)$, where

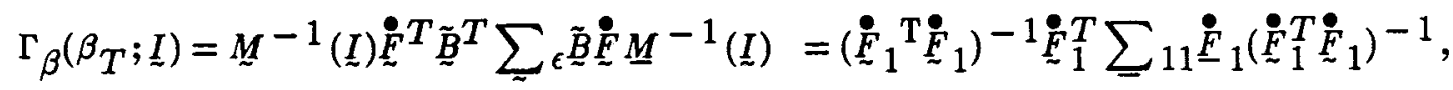

$\sum_{\epsilon}=\left[\begin{array}{ll}\sum_{11} & \sum_{12} \\ \sum_{12}^{T} & \sum_{22}\end{array}\right]$ and $\sum_{11}$ is $v \times v$.

It is remarkable in Corollary 2.6 that the asymptotic covariance matrix of the ols estimator $\underline{\hat{\beta}} I N$ is the same as it would be if the "recapture" data were left out of the $\underline{Y}$ - vector in (2.5)! (The residual variance matrix $\sum_{11}$ involves only the $\underline{Y}_{1}$ component of $\left.\underline{Y}\right)$. of course this covariance 
matrix applies only as the matrix $\underset{\tilde{B}}{\vec{N}} \underset{\tilde{B}}{\underline{B}}$ (i.e. as the ratio $g_{N} / n_{N} \rightarrow 0$ ), which, for all the $n_{t}$ 's being equal, is the same as the sampling fraction $(S F)$ $n_{t} / N \rightarrow 0$. In fact, the Monte Carlo results in Section 2.8 show that for $S F$ ' $s$ of $5 \%, 10 \%$ or $20 \%$, the ols estimate $\hat{\beta}_{I N}$ dramatically out-performs the "norecaptures" estimate. This may be attributed to the $S F$ being too far from zero for the limit result of Corollary 2.6 to apply. What's more, we found that the covariance matrix of $\underline{\hat{\beta}}_{I N}$ is much better estimated by replacing $\underline{\tilde{B}}$ by $\tilde{\sim}_{N}$ in Corollary 2.6 than by using the final expression which omits $\underset{B}{\tilde{B}}$. Interestingly, the asymptotic convariance matrix of the optimal gls estimator $\underline{\widehat{\beta}} N$ in Corollary 2.5 depends on the recapture process only through the matrix $\sum_{11}^{*}$, which by a partitioned matrix inverse formula satisfies

$\sum_{11}^{*}=\left(\sum_{11}-\sum_{12} \sum_{22}^{-1} \sum_{12}^{T}\right)^{-1}$

Of course, $\sum_{11}^{*}$ is the inverse of the conditional covariance matrix of the prediction error by the marginal component ${\underset{\sim}{1}}_{1}$, given the recapture data $\underline{Y}_{2}$. Although this observation may have some intuitive merit, it was again found that a better estimate of the covariance matrix of $\underline{\hat{\beta}}_{N}$ was obtained by replacing $\tilde{B}$ by $\tilde{B}_{N}$ in Corollary 2.5 .

\subsubsection{Estimating $\sum_{\epsilon}$}

The asymptotically optimal weight matrix $\sum_{\epsilon}$ is given in Lemma 2.3 as

$\left.\sum_{\epsilon}=\stackrel{\bullet}{\xi}\left(W^{*}\right) \underline{L} \underline{\sim}_{W} L^{T} \underline{\xi}^{\bullet} T_{(}{ }^{*}\right)$. Here $\underline{\Gamma}_{W}$ is the asymptotic covariance matrix of $\underline{B}_{N}\left\{\underline{W}_{N}-W^{*}\right\}$. For estimating $\sum_{\epsilon}$ we shall therefore replace $\Gamma_{W}$ by the matrix $\underline{\sim}_{N} \operatorname{Var}\left(\underline{W}_{N}\right) \underline{B}_{N}$, which itself must be estimated. Natural estimates of $L$ and $\dot{\xi}\left(\underline{w}^{*}\right)$ are obtained by replacing $\lambda_{t}$ by $n_{\mathrm{t}} / n_{N}$ in $\underline{L}$, and by replacing $q^{*}$ by $\widehat{q}_{N}^{*}$ and ${\underset{\sim}{\beta}}_{T}$ by $\widehat{\beta}_{I N}$ ( the consistent ols estimate) in $\stackrel{\dot{\xi}}{\underline{\alpha}}\left(w^{*}\right)$.

Now the matrix $\operatorname{Var}\left(\underline{W}_{N}\right)$ has the form $\operatorname{Var}\left(\underline{W}_{N}\right)=\left[\begin{array}{cc}\operatorname{var}\left(\underline{\widehat{q}}_{N}^{*}\right) & \operatorname{cov}\left(\underline{\hat{q}}_{N}^{*}, \underline{Y}_{2 N}\right) \\ \operatorname{cov}^{T}\left(\underline{\widehat{q}}_{N}^{*}, \underline{Y}_{2 N}\right) & \operatorname{var}\left(\underline{Y}_{2 N}\right)\end{array}\right]$. 
The matrix $\operatorname{var}\left(\widehat{\widehat{q}}_{N}^{*}\right)$ is given following (2.17) with a consistent estimate provided by $(2.17)$.

The derivations of $\operatorname{Var}\left(\underline{Y}_{2 N}\right)$ and $\operatorname{cov}\left(\underline{\widehat{q}}_{N}^{*}, \underline{Y}_{2 N}\right)$ are long and tedious but straight-forward, so only the basic ideas and final results are given here, along with the basic idea for obtaining consistent estimates. Complete details are in HHE.

$\operatorname{Var}\left(\underline{Y}_{2}\right)=\operatorname{Var}\left[\begin{array}{c}\hat{p}_{1} \\ \cdots \\ \hat{p}_{m}\end{array}\right]$ is a matrix of $(m-1) \times(m-1)$ blocks, where the

$(i, j)$ block is $\operatorname{cov}\left(\underline{\widehat{p}}_{i}, \underline{\hat{p}}_{j}\right), 1 \leq i, j \leq m$.

Now for $1 \leq i \leq m, \underline{\hat{p}}_{i}=\underline{R}_{i} / D_{i}$ is a ratio of random quantities. If we define $g: \mathbb{R}^{m} \rightarrow \mathbf{R}^{m-1}$ by $\left.g\left(x_{1}, \cdots, x_{m}\right)=\left[g_{1}(x), \cdots, g_{m-1}(x)\right)\right]^{T}=\left[x_{1} / x_{m}, \cdots, x_{m-1} / x_{m}\right]^{T}$, then $\underline{\hat{p}}_{i}=\underline{g}\left(\underline{R}_{i}, D_{i}\right)$, so we can use a delta method approximation. Writing $\underline{J}_{g}$ as the Jacobian of $g$ and setting $\underline{\mu}_{i}=E\left[\underline{R}_{i}^{T}, D_{i}\right]$, we have

$\operatorname{Cov}\left(\underline{\hat{p}}_{i}, \underline{\widehat{p}}_{j}\right)=E\left\{\left[\underline{\widehat{\hat{p}}}_{i}-E \underline{\widehat{p}}_{i}\right]\left[\underline{\hat{p}}_{j}-E \underline{\hat{p}}_{j}\right]^{T}\right\} \approx E\left\{\left[\underline{g}_{i}\left(\underline{R}_{i}, D_{i}\right)-\underline{g}_{i}\left(\underline{\mu}_{i}\right)\right]\left[\underline{g}\left(\underline{R}_{j}, D_{j}\right)-\underline{g}(\underline{\mu} j)\right]^{T}\right\}$

$\left.\approx E\left\{\underline{J}_{g}\left(\underline{\mu}_{i}\right)\left[\begin{array}{c}\underline{R}_{i}-E \underline{R}_{i} \\ D_{i}-E D_{i}\end{array}\right]\right\}\left\{\underline{J}_{g}\left(\underline{\mu}_{j}\right)\left[\begin{array}{c}\underline{R}_{j}-E \underline{R}_{j} \\ D_{j}-E D_{j}\end{array}\right]\right\}^{T}=\underline{J}_{g}\left(\underline{\mu}_{i}\right) \underline{W}_{i j} \underline{J}_{g}^{T} \underline{\mu}_{j}\right)$,

where

$$
\underline{W}_{i j}=\left[\begin{array}{cc}
\operatorname{cov}\left(\underline{R}_{i}, \underline{R}_{j}\right) & \operatorname{cov}\left(\underline{R}_{i}, D_{j}\right) \\
\operatorname{cov}\left(D_{i}, \underline{R}_{j}\right) & \operatorname{cov}\left(D_{i}, D_{j}\right)
\end{array}\right] .
$$

Thus, our approximation to $\operatorname{Var}\left(\underline{Y}_{2}\right)$ has $(i, j)$ block $\underline{J}_{g}\left(\underline{\mu}_{i}\right) \underline{W}_{i j} \underline{J}_{g}^{T}\left(\underline{\mu}_{j}\right)$. We need on ly compute $\underline{\mu}_{i}$ and $\underline{W}_{i j}$.

In this direction let $\underline{c}_{i l t}=: Z_{l t i} \underline{Z}_{l, t+1}$, so that

$\underline{R}_{i}=\sum_{t=1}^{r} g^{1} \bar{N}_{t}^{1} \sum_{l=1}^{N} \underline{c}_{i l t} I_{l t} I_{l, t+1}$. Then straight-forward calculation gives 
$E\left(\underline{R}_{i}\right)=\sum_{t} g_{N_{t}}^{-1} \sum_{l} \underline{c}_{i l t} E\left(I_{l t} I_{l, t+1}\right)=\sum_{t} g_{N_{t}}^{-1} \sum_{l} \underline{c}_{i l t} e_{t}=\sum_{t=1}^{r-1} \bar{c}_{i t}^{(N)}$

where $e_{t} \equiv f_{t} f_{t+1}$ and $\overline{\underline{c}}_{i t}^{(N)}=: \frac{1}{N} \sum_{l=1}^{N} \underline{c}_{i l t}$, and

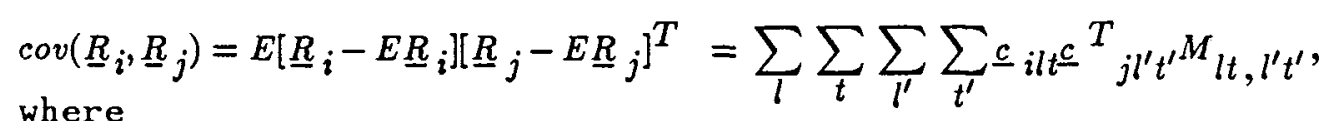

$g_{N t^{g}} N t^{M} M_{l t, l^{\prime} t^{\prime}}=$

$l=l^{\prime}, t=t^{\prime}: e_{t}\left(1-e_{t}\right)$

$l \neq l^{\prime}, t=t^{\prime}: \quad \frac{n_{t}\left(n_{t}-1\right)}{N(N-1)} \quad \frac{n_{t+1}\left(n_{t+1}-1\right)}{N(N-1)}-e_{t}^{2}$

$\left|t-t^{\prime}\right|>1$, all $l, l^{\prime}: 0$

$t^{\prime}=t+1, l=l^{\prime}: \frac{n_{t}}{N} \quad \frac{n_{t+1}}{N} \frac{n_{t+2}}{N}-e_{t} e_{t+1}$

$t^{\prime}=t+1, \quad l \neq l^{\prime}: \frac{n_{t}}{N} \frac{n_{t+1}\left(n_{t+1}-1\right)}{N(N-1)} \frac{n_{t+2}}{N}-e_{t} e_{t+1}$

$t^{\prime}=t-1, l=l^{\prime}: \frac{n_{t}}{N} \frac{n_{t+1}}{N} \frac{n_{t-1}}{N}-e_{t} e_{t-1}$

$t^{\prime}=t-1, l \neq l^{\prime}: \frac{n_{t}\left(n_{t}-1\right)}{N(N-1)} \cdot \frac{n_{t+1}}{N} \cdot \frac{n_{t-1}}{N}-e_{t} e_{t-1}$.

Similarly, defining $\underline{D}=\left[D_{1}, \cdots, D_{m}\right]^{T}$ and $\bar{Z}_{t}^{o(N)}=\frac{1}{N} \sum_{l=1}^{N} Z_{l t}^{o}$,

$\underline{D}=\sum_{t=1}^{r} \sum^{-1}-1, \sum_{l=1}^{N} \underline{Z}_{l t}^{0} I_{l t} I_{l, t+1}$, so

$E(\underline{D})=\sum_{t=1}^{r} \bar{\sum}^{1} g_{N t}^{-1} \sum_{l=1}^{N} \underline{Z}_{l t}^{0} E\left[I_{l t} I_{l, t+1}\right]=\sum_{t} g_{N t}^{-1} \sum_{l} \underline{Z}_{l t^{e} t}^{0}=\sum_{t=1}^{r-1} \underline{Z}_{t}^{0(N)}$

and $\operatorname{cov}\left(D_{i}, D_{j}\right)$ is the $(i, j)$ element of 
$\operatorname{Var}(\underline{D})=E\left\{[\underline{D}-E \underline{D}][\underline{D}-E \underline{D}]^{T}\right\}=\sum_{t} \sum_{l} \sum_{t^{\prime}} \sum_{l^{\prime}} \underline{Z}_{l t}^{0} \underline{Z}_{l^{\prime} t^{\prime}}^{0 T} M_{l t^{\prime} l^{\prime} t^{\prime}}$.

Finally, the matrices $\operatorname{cov}\left(D_{i}, \underline{R}_{j}\right)$ are submatrices of

$\operatorname{cov}\left(\underline{D}, \underline{R}_{j}\right)=E\left\{[\underline{D}-E \underline{D}]\left[\underline{R}_{j}-E \underline{R}_{j}\right]^{T}\right\}=\sum_{t} \sum_{l} \sum_{t^{\prime}} \sum_{l^{\prime}} \underline{Z}_{l t^{\prime}}^{0}{\underline{j l^{\prime}}}_{t^{\prime}}^{T} M_{l t, l^{\prime} t^{\prime}}$.

From (2.8) and (2.9), the $(r-1)(m-1) \times m(m-1)$ matrix

$\operatorname{cov}\left(\underline{Y}_{1}, \underline{Y}_{2}\right)=\operatorname{cov}\left(\underline{A}^{*} \underline{\underline{q}}^{*}, \underline{Y}_{2}\right)=\underline{A} \operatorname{cov}\left(\underline{\widehat{q}}^{*}, \underline{Y}_{2}\right)$

and

$\operatorname{cov}\left(\underline{\widehat{q}}^{*}, \underline{Y}_{2}\right)=E\left\{\left[\underline{\hat{q}}^{*}-E \underline{\hat{q}}^{*}\right]\left[\underline{Y}_{2}-E \underline{Y}_{2}\right]^{T}\right\}$

has block structure with $(t, i)$ block $(1 \leq t \leq r, 1 \leq i \leq m)$

$\underline{B}_{t i}=: \operatorname{cov}\left(\underline{\widehat{q}}_{t}, \underline{\hat{p}}_{i}\right)=E\left\{\left[\underline{\hat{q}}_{t}-E \underline{\hat{q}}_{t}\right]\left[\underline{\hat{p}}_{i}-E \underline{\hat{p}}_{i}\right]^{T}\right\} \approx E\left\{\left[\underline{\hat{q}}_{t}-E \underline{\underline{q}}_{t}\right]\left[g\left(\underline{R}_{i}, D_{i}\right)-g\left(\underline{\mu}_{i}\right)\right]^{T}\right\}$

$$
\begin{aligned}
& \approx E\left[\left\{\underline{\hat{q}}_{t}-E \underline{\hat{q}}_{t}\right\}\left\{\underline{J}_{g}\left(\underline{\mu}_{i}\right)\left[\begin{array}{c}
\underline{R}_{i}-E \underline{R}_{i} \\
D_{i}-E D_{i}
\end{array}\right]\right\}^{T}\right] \\
& =E\left\{\left[\underline{\hat{q}}_{t}-E \underline{\underline{q}}_{t}\right]\left[\left(\underline{R}_{i}-E \underline{R}_{i}\right)^{T}, D_{i}-E D_{i}\right]\right\} \underline{J}_{g}^{T}\left(\underline{\mu}_{i}\right) \\
& =\left[\operatorname{cov}\left(\underline{\hat{q}}_{t}, \underline{R}_{i}\right) \vdots \operatorname{cov}\left(\underline{\hat{q}}_{t}, D_{i}\right)\right] \underline{J}_{g}^{T}\left(\underline{\mu}_{i}\right)=\left[\underline{H}_{t i}^{(1)} \quad \vdots \quad \underline{H}_{t i}^{(2)}\right] \underline{J}_{g}^{T}\left(\underline{\mu}_{i}\right), \text { say } .
\end{aligned}
$$

Now since $\underline{\widehat{q}}_{t}=\left[\underline{I}_{m-1}: \underline{Q}_{m-1}\right] \underline{\hat{q}}_{t}^{0}=: \underline{B}_{\underline{\hat{q}}}^{0}$,

$\underline{H}_{t i}^{(1)}=\operatorname{cov}\left(\underline{B}_{\hat{q}}^{0}, \underline{R}_{i}\right)=\underline{B} \operatorname{cov}\left(\underline{\widehat{q}}_{t}^{0}, \underline{R}_{i}\right)=\underline{B}_{t i}^{0}$,

say, where for fixed $t \in\{1, \cdots, r-1\}$,

$\underline{G}_{t i}^{0}=: E\left\{\left[\underline{\underline{q}}_{t}^{0}-\underline{E} \underline{q}_{t}^{0}\right]\left[\underline{R}_{i}-E \underline{R}_{i}\right]^{T}\right\}$ 
$=\frac{1}{n_{t}} \sum_{l=1}^{N} \sum_{l^{\prime}=1}^{N}\left\{g_{N t}^{-1} E_{t l l^{\prime}}^{*} \underline{Z}_{l t}^{0} \underline{c} \underset{i l^{\prime} t}{T}+g_{\bar{N}, t-1}^{-1} I(t-1 \geq 1) E_{t l^{\prime}}^{* *} \underline{Z}_{l t}^{0} \underline{c}_{i l^{\prime}, t-1}^{T}\right\}$.

where

$$
\begin{aligned}
E_{t l l^{\prime}}^{*}= & \text { if } l=l^{\prime}: e_{t}\left(1-f_{t}\right) \\
& \text { if } l \neq l^{\prime}: e_{t}\left[\frac{n_{t}-N}{N(N-1)}\right]
\end{aligned}
$$

and

$$
\begin{aligned}
E_{t l^{\prime}}^{* *}= & \text { if } l=l^{\prime}: e_{t-1}\left(1-f_{t}\right) \\
& \text { if } l \neq l^{\prime}: e_{t-1}\left[\frac{n_{t}-N}{N(N-1)}\right] .
\end{aligned}
$$

Now define $e_{m i}$ to be the column vector in $\mathbb{R}^{m}$ with entries all zero except position $i$, which is one. Then for $\underline{H}_{t i}^{(2)}$ in $(2.23)$, we have $\underline{H}_{t i}^{(2)}=$ $\operatorname{cov}\left(\underline{\widehat{q}}_{t}, D_{i}\right)=\operatorname{cov}\left(\underline{B} \underline{\hat{q}}_{t}^{0}, \underline{e}_{m i}^{T} \underline{T}\right)$

$=\underline{B} \operatorname{cov}\left(\underline{\hat{q}}_{t}^{0}, \underline{D}\right) \underline{e}_{m i}$ and

$$
\operatorname{cov}\left(\underline{\widehat{q}}_{t}^{0}, \underline{D}\right)=E\left\{\left[\underline{\widehat{q}}_{t}^{0}-E \underline{\widehat{q}}_{t}^{0}\right][\underline{D}-E \underline{D}]^{T}\right\}
$$

$=\frac{1}{n_{t}} \sum_{l} \sum_{l^{\prime}}\left\{g_{N_{t}}^{1} E_{t l l^{\prime}}^{*} \underline{Z}_{l t}^{0} \underline{Z}_{l^{\prime} t}^{0 T}+g \bar{N}_{N, t-1}^{1} I(t-1 \geq 1) E_{t l l^{\prime}}^{* *} \underline{Z}_{l t}^{0} \underline{Z}_{l^{\prime}, t-1}^{0 T}\right\}$.

Consistent estimation of $\sum_{\epsilon}$ comes down to consistent estimation of $\operatorname{Var}\left(\underline{W}_{N}\right)$, which reduces to such for $\operatorname{Var}\left(\underline{Y}_{2 N}\right)$ and $\operatorname{cov}\left(\underline{\widehat{q}}_{N}^{*}, \underline{Y}_{2 N}\right)$ since $\operatorname{Var}\left(\underline{\widehat{q}}_{N}^{*}\right)$ is consistently estimated by $(2.17) . \quad \operatorname{Var}\left(\underline{Y}_{2 N}\right)$ and $\operatorname{cov}\left(\underline{\widehat{q}}_{N}^{*}, \underline{Y}_{2 N}\right)$ can be consistently estimated, under model (2.4), via the following approach. The basic idea is to express the variance formulae in terms of certain population fractions which, under model (2.4), have representations in terms of the marginal probabilities $q_{t i}$ and the transition probabilities $p_{i j}$. Then $\widehat{q}_{t i}$ and the consistent ols estimate $\hat{p}_{i j}=p_{i j}\left(\widehat{\beta}_{I}\right)$ may be plugged into the resulting expressions to yield consistent estimates.

To estimate $\operatorname{Var}\left(\underline{Y}_{2}\right)$ it is clearly enough to estimate $\underline{J}_{g}\left(\underline{\mu}_{i}\right)$ and $\underline{W}_{i j}$ 
given by $(2.22)$. A consistent estimate of $\underline{J}_{g}\left(\underline{\mu}_{i}\right)$ is $\underline{J}_{g}\left(\underline{\underline{\mu}}_{i}\right)$ where $\underline{\hat{\mu}}_{i}^{T}=$ $\left[\underline{\widehat{R}}_{i}, \hat{D}_{i}\right]$ and $\underline{\widehat{R}}_{i}$ and $\widehat{D}_{i}$ are defined as $\underline{R}_{i}$ and $D_{i}$ with $g_{N t}$ replaced by $n_{t, t+1}$. For $\underline{W}_{i j}$, all four of the component submatrices depend on the constants $M_{l t^{\prime} l^{\prime} t^{\prime}}$ which depend on the sampling fractions $f_{t}$ at each wave $t$. Since these are usually known only roughly, a simple approximation $\widehat{M}_{l t, l^{\prime} t^{\prime}}$ to $M_{l t, l^{\prime} t^{\prime}}$ is obtained by replacing (ala assumption (A3)) terms like $\left(n_{t}-1\right) /(N-1)$ by $f_{t}$, so that

$$
\begin{array}{llll}
g_{N t} g_{N t^{\prime}} \widehat{M}_{l t, l^{\prime} t^{\prime}}= & l=l^{\prime}, & t=t^{\prime}: & e_{t}\left(1-e_{t}\right) \\
& l \neq l^{\prime}, & t=t^{\prime}: & 0 \\
& a 11 \quad l, l^{\prime}, & \left|t-t^{\prime}\right|>1: & 0 \\
& l=l^{\prime}, & t^{\prime}=t+1: & e_{t}\left(f_{t+2}-e_{t+1}\right) \\
& l \neq l^{\prime}, & t^{\prime}=t+1: & 0 \\
l=l^{\prime} & t^{\prime}=t-1: & e_{t}\left(f_{t-1}-e_{t-1}\right) \\
l \neq l^{\prime}, & t^{\prime}=t-1: & 0
\end{array}
$$

The upper left submatrix of $\underline{W}_{i j}$, with $M_{l t}, l^{\prime} t^{\prime}$ replaced by $\widehat{M}_{l t^{\prime}} l^{\prime} t^{\prime}$, is given by

$$
\begin{aligned}
& \sum_{l=1}^{N} \sum_{t=1}^{r} \sum_{l^{\prime}=1}^{N} \sum_{t^{\prime}=1}^{r-1} \underline{c}_{i l t} \underline{c}_{j l^{\prime} t^{\prime}}^{T} \widehat{M}_{l t, l^{\prime} t^{\prime}} \\
& =\sum_{t=1}^{r=1} \sum_{t^{\prime}=1}^{r-1} \sum_{l=1}^{N} \hat{M}_{l t, l t^{\prime}} \underline{c}_{i l t^{c}}{\stackrel{T}{j l t^{\prime}}}^{T} \\
& =\sum_{t=1}^{r} \sum_{l=1}^{N} \hat{M}_{l t, l t} \underline{c}_{i l t} \underline{\underline{c}} \underset{j l t}{T}+\sum_{t \neq t^{\prime}} \sum_{l=1}^{N} \hat{M}_{l t, l t^{\prime} \underline{c}_{i l t}}{\stackrel{\mathcal{c}}{j l t^{\prime}}}^{T} \\
& =\sum_{t=1}^{r-1} \sum_{l=1}^{N} \widehat{M}_{l t, l t} \underline{c}_{i l t} \underline{c}_{j l t}^{T} \\
& +\sum_{t=1}^{r-2} \sum_{l=1}^{N} \widehat{M}_{l t, l, t+1}\left\{_{i l t} \underline{c}_{j l, t+1}^{T}+\underline{c}_{i l, t+1} \underline{c}_{j l t}^{T}\right\},
\end{aligned}
$$

the third equality following since $\widehat{M}_{l t, l t^{\prime}}=\widehat{M}_{l t^{\prime}, l t}$ for $t \neq t^{\prime}$ and $\widehat{M}_{l t, l t^{\prime}}=0$ if $\left|t-t^{\prime}\right|>1$. Toward consistent estimates of (2.24) and (2.25) by the above- 
noted scheme, note that $\widehat{M}_{l t, l t^{\prime}}$ does not depend on $l$, so define $d_{N t t^{\prime}}=N \widehat{M}_{l t, l t^{\prime}}$. Then (2.24) equals (writing $\delta_{i j}$ as the Kronecker delta)

$$
\begin{aligned}
& \sum_{t=1}^{r} d_{N t t}\left\{\frac{1}{N} \sum_{l=1}^{N} Z_{l t i} Z_{l t j} Z_{l, t+1} Z_{l, t+1}^{T}\right\} \\
& =\delta_{i j} \sum_{t=1}^{r-1} d_{N t t}\left\{\frac{1}{N} \sum_{l=1}^{N} Z_{l t i} Z_{l, t+1} Z_{l, t+1}^{T}\right\} \\
& =\delta_{i j} \sum_{t=1}^{r-1} d_{N t t} D i a g\left\{\tau_{i 1}^{t}, \cdots, \tau_{i, m-1}^{t}\right\},
\end{aligned}
$$

where $\tau_{i k}^{t}=$ fraction of the population in stage $i$ at wave $t$ and in stage $k$ at wave $t+1$. Under the Markov model (2.4), $\tau_{i k}^{t}=q_{t i} p_{i k}$, so the above-noted method gives a consistent estimate of (2.24). Similarly (2.25) may be shown to equal $\sum_{t=1}^{r-2} d_{N t, t+1}\left\{e_{m-1},{ }_{j}\left[\alpha_{i j 1}^{t}, \cdots, \alpha_{i j, m-1}^{t}\right]^{T}+\left[\alpha_{j i 1}^{t}, \cdots, \alpha_{j i, m-1}^{t}\right]^{T} e_{m-1, i}^{T}\right\}$, where $\alpha_{i k j}^{t}=$ fraction of the population in stage $i$ at wave $t$, in stage $k$ at wave $t+1$ and in stage $j$ at wave $t+2$. Under the Markov model (2.4), $\alpha_{i k j}^{t}=q_{t i} p_{i k} p_{k j}$, so the proposed scheme provides a consistent estimate of $(2.25)$.

The corresponding representations of $\operatorname{cov}\left(\underline{R}_{i}, D_{j}\right)$ and $\operatorname{cov}\left(D_{i}, D_{j}\right)$ in $(2.22)$ are

$$
\begin{aligned}
& \operatorname{cov}\left(\underline{R}_{i}, D_{j}\right) \approx \delta_{i j} \sum_{t=1}^{r-1} d_{N t t}\left[\tau_{i 1}^{t}, \cdots, \tau_{i, m-1}^{t}\right]^{T} \\
& +\sum_{t=1}^{r-2} d_{N t, t+1}\left\{\left[\begin{array}{ccc}
\alpha_{1 i 1}^{t} & \cdots & \alpha_{m i 1}^{t} \\
\vdots & & \vdots \\
\alpha_{1 i, m-1}^{t} & \alpha_{m i, m-1}^{t}
\end{array}\right]+\left[\begin{array}{ccc}
\tau_{i 1}^{t} & 0 & 0 \\
& \ddots & \vdots \\
0 & \tau_{i, m-1}^{t} & 0
\end{array}\right]\right\} \underline{e}_{m j}, \\
& \operatorname{cov}\left(D_{i}, D_{j}\right) \approx \delta_{i j} \sum_{t=1}^{r-1} d_{N t t} q_{t i}+2 \sum_{t=1}^{r-2} d_{N t, t+1} \tau_{i j}^{t} .
\end{aligned}
$$

These results provide the consistent estimate of $\underline{W}_{i j}$ in (2.22), and hence for $\operatorname{Var}\left(\underline{Y}_{2}\right)$.

To obtain a consistent estimate of $\operatorname{cov}\left(\underline{Y}_{1}, \underline{Y}_{2}\right)$, it is enough (by (2.23) and the ensuing computations) to estimate $G_{t i}^{o}$ and $\operatorname{cov}\left(\underline{\underline{q}}_{t}^{o}, \underline{D}\right)$. But these may be expressed (with approximations $\widehat{E}_{t l^{\prime}}^{*}=\delta_{l l^{\prime}} e_{t}\left(1-f_{t}\right)$ and $\widehat{E}_{t l^{\prime}}^{* *}=\delta_{l l^{\prime}} e_{t-1}\left(1-f_{t}\right)$ substituted for $E_{t l l^{\prime}}^{*}$ and $E_{t l l^{\prime}}^{* *}$ ) as 


$$
\begin{aligned}
& G_{t i}^{o} \approx n_{t}^{-1}\left(1-f_{t}\right)\left\{I(\leq r-1) \underline{e}_{m i} q_{t i}\left[p_{i 1}, \cdots, p_{i, m-1}\right]\right. \\
& \left.+I(t \geq 2) q_{t-1, i}\left[\begin{array}{ccc}
p_{i 1} & \ddots & 0 \\
0 & p_{i, m-1} \\
-0 & \cdots & 0
\end{array}\right]\right\}, \\
& \operatorname{cov}\left(\underline{\underline{q}}_{t}^{o}, D\right)=n_{t}^{-1}\left(1-f_{t}\right)\left\{\operatorname{Diag}\left(\underline{q}_{t}^{o}\right)+I(t \geq 2) P^{T} \operatorname{Diag}\left(\underline{q}_{t-1}^{o}\right)\right\} .
\end{aligned}
$$

So the proposed method works here as well.

Unfortunately, $\hat{\sum} \epsilon$ may not be p.d. in general, due to the piece-meal construction of $\operatorname{Var}\left(\underline{W}_{N}\right)$. This causes problems in implementing our method on a nonlinear regression package which only does ols calculations, since one needs to obtain the square root of $\hat{\sum} \epsilon^{-1}$ to do generalized least squares via ols in the transformed model. We remedied this problem by replacing $\hat{\sum}_{\epsilon}$ by the p.d. matrix $\tilde{\sum}_{\epsilon}=\left(\hat{\sum}_{\epsilon}^{T} \hat{\sum_{\epsilon}}\right)^{1 / 2}$, where the square root is the eigen-square-root. This seems justifiable since $\tilde{\sum} \epsilon$ has the same plimit as does $\hat{\sum} \epsilon$.

\subsection{Lack of Fit Testing}

It follows from Corollary 2.5 and standard theory that, with $\underline{Q}=\tilde{\sum} \bar{\epsilon}^{-1}$ and $\underline{\hat{\beta}} G L S$ denoting the corresponding estimator of $\underline{\beta}$, $\mathrm{X}_{L O F}^{2}=: n_{N} S_{N}(\underline{\hat{\beta}} G L S)$ has approximately the $\chi_{s-v}^{2}$ distribution under model (2.4), where $s=\operatorname{dim}(\underline{\sim})=(r+m-1)(m-1)$ and $v=\operatorname{dim}(\underline{\beta})=m(m-1)$. This statistic is, of course, just the value of the error sum of squares from the generalized least squares fit of (2.5) using $Q=\tilde{\sum}_{\epsilon}^{-1}$, so it is easily obtained.

\subsection{Some Remarks on Computing}

Computation of the asymptotically optimal $\underline{\hat{\beta}}_{N}$ and its covariance matrix in Corollary 2.5 requires

1) the ols estimate $\underline{\widehat{\beta}} I N$

2) the estimated optimal weight matrix $\dot{\sum}_{\epsilon}$.

The ols estimate $\underline{\hat{\beta}} I$ can be obtained by any standard nonlinear least squares program. We used SAS's NLIN procedure. The estimate $\tilde{\sum} \varepsilon$ requires 
considerable pre-processing of the basic data estimates. We used SAS's IML procedure for this. Given $\sum_{\epsilon}$, we transformed model (2.5) by multiplication by the inverse of the eigen-square-root of $\tilde{\sum_{\epsilon}}$, and then fit the resulting model using NLIN. By this method, the estimate given by NLIN equals $\underline{\widehat{\beta}}_{G L S}$, and the covariance estimate computed by NLIN equals the estimated covariance matrix in Corollary 2.5. Also, the lack of fit statistic $X_{L O F}^{2}$ comes out naturally as the error sum of squares. The IML procedure is then used following NLIN to compute the GLS estimate $\underline{\hat{p}}^{(G L S)}$ of the $p_{i j}$ 's via (2.10), along with their delta method covariance estimate. A SAS program to do these computations is available from the first author.

\subsection{Wonte Carlo Study}

To verify the asymptotic results and to investigate the sensitivity of the performance of the estimates to the sampling fraction, a Monte Carlo study was performed. The design parameters were: $m=4$ categories for $Z$ and $r=8$ sampling waves, initial distribution $\underline{q}_{1}^{O}=(.80, .10, .05, .05)^{T}$ and transition matrix

$$
\stackrel{P}{P}=\left[\begin{array}{llll}
.80 & .10 & .05 & .05 \\
.05 & .50 & .40 & .05 \\
.05 & .05 & .50 & .40 \\
.05 & .05 & .20 & .70
\end{array}\right]
$$

Table 2.3(a) shows the results of 100 Monte Carlo replications for population size $N=1000$ and constant (from wave to wave) sampling fractions of $10 \%$ and $20 \%$. Tabel $2.3(\mathrm{~b})$ shows similar results for $N=10,000$ and sampling fractions of $5 \%$ and $10 \%$. Displayed in these tables are the gls and ols results for estimating $\underset{\sim}{P}$ using the haphazard recapture data, and also (in the column headed "No Recap") the results of ols estimation (analogous to Kabfleisch \& Lawless (1984)) which does not use the recapture data. The columns headed "Ave Est" and "MC se" are the average and standard deviation of the 100 Monte Carlo estimates. The column headed "Est se" is the square root of the average variance estimate, obtained from the asymptotic variance matrix of $\underline{\widehat{\beta}}$ for the given method and subsequent application of the delta method. 
Looking at the Monte Carlo results, one sees that the gls estimator only modestly out-performs the ols estimator (of course this depends on $\underline{q}_{1}^{o}$ and $\underline{P})$, but both of these estimators dramatically improve on the no-recaptures estimator. Also, the estimated standard errors are sufficiently close to the Monte Carlo standard error estimates to be reliably used for standard "normal theory" inferences.

Table 2.3 goes here 
able 2.3. Monte Carlo Results for Estimating the Transition Matrix

$N=1,000$

\begin{tabular}{cccccc}
$s f=10 \%$ & \multicolumn{3}{c}{$s f=20 \%$} \\
\hline GLS & OLS No recap. & GLS & OLS Recap.
\end{tabular}

\begin{tabular}{|c|c|c|c|c|c|c|c|c|c|c|c|c|c|c|c|c|c|}
\hline \multicolumn{2}{|c|}{$\overline{\text { Param True }}$} & $\begin{array}{l}\text { Ave } \\
\text { Est }\end{array}$ & $\begin{array}{l}\text { MC } \\
\text { s.e.* }\end{array}$ & $\begin{array}{l}\overline{\text { Est }} \\
\text { s.e.* }\end{array}$ & $\begin{array}{l}\text { Ave } \\
\text { Est. }\end{array}$ & $\begin{array}{l}\mathrm{MC} \\
\text { s.e.* }\end{array}$ & $\begin{array}{l}\text { Est. } \\
\text { s.e.* }\end{array}$ & $\begin{array}{l}\text { Ave } \\
\text { Est. }\end{array}$ & $\begin{array}{l}\text { MC } \\
\text { s.e. }^{*}\end{array}$ & $\begin{array}{l}\text { Ave. } \\
\text { Est. }\end{array}$ & $\begin{array}{l}\text { mc } \\
\text { s.e.* }\end{array}$ & $\begin{array}{l}\text { Est. } \\
\text { s.e." }\end{array}$ & $\begin{array}{l}\text { Ave. } \\
\text { Est. }\end{array}$ & $\begin{array}{l}\text { MC } \\
\text { s.e." }\end{array}$ & $\begin{array}{l}\text { Est } \\
\text { s.e.* }\end{array}$ & $\begin{array}{l}\text { Ave } \\
\text { Est. }\end{array}$ & $\begin{array}{l}\text { me } \\
\text { s.e.* }\end{array}$ \\
\hline$p_{11}$ & .80 & .80 & 2.9 & 3.1 & .78 & $\mathbf{3 . 3}$ & 3.8 & .74 & 6.7 & .80 & 1.5 & 1.6 & .80 & 1.9 & 2.0 & .76 & 3.4 \\
\hline$p_{12}$ & .10 & .10 & 2.7 & 2.9 & .10 & 2.9 & 3.7 & .14 & 3.8 & .10 & 1.3 & 1.5 & .10 & 1.6 & 1.8 & .14 & 3.3 \\
\hline$p_{13}$ & .05 & .05 & 2.9 & 2.7 & .06 & 3.1 & 3.9 & .06 & 4.8 & .05 & 1.5 & 1.4 & .06 & 1.7 & 1.9 & .07 & 4.2 \\
\hline$p_{21}$ & .05 & .06 & 7.2 & 9.1 & .07 & 8.1 & 11.1 & .27 & 35.4 & .03 & 3.8 & 3.6 & .05 & 4.1 & 4.0 & .23 & 28.6 \\
\hline$p_{22}$ & .50 & .44 & 11.9 & 13.3 & .46 & 13.8 & 19.1 & .09 & 15.1 & .49 & 6.5 & 6.2 & .50 & 7.3 & 8.2 & .11 & 16.0 \\
\hline$p_{23}$ & .40 & .44 & 14.1 & 13.8 & .39 & 14.8 & 18.7 & .43 & 33.2 & .42 & 6.5 & 6.7 & .40 & 7.4 & 8.1 & .37 & 33.1 \\
\hline$p_{31}$ & .05 & .05 & 5.6 & 6.7 & .07 & 6.2 & 9.0 & .07 & 12.3 & .03 & 2.7 & 2.9 & .05 & 3.0 & 3.3 & .05 & 9.6 \\
\hline$p_{32}$ & .05 & .06 & 6.1 & 6.7 & .06 & 5.4 & 8.1 & .15 & 16.8 & .03 & 2.7 & 2.8 & .05 & 2.9 & 3.1 & .19 & 17.3 \\
\hline$P_{33}$ & .50 & .45 & 12.2 & 11.7 & .46 & 11.5 & 13.9 & .25 & 24.7 & .50 & 5.9 & 5.3 & .49 & 6.1 & 5.9 & .25 & 24.4 \\
\hline$p_{41}$ & .05 & .06 & 5.3 & 5.9 & .07 & 6.2 & 7.9 & .06 & 9.4 & .03 & 2.4 & 2.7 & .08 & 3.0 & 3.2 & .04 & 6.0 \\
\hline$p_{42}$ & .05 & .07 & 3.4 & 5.7 & .06 & 3.1 & 7.0 & .12 & 11.4 & .05 & 2.4 & 2.6 & .05 & 2.7 & 2.9 & .09 & 10.9 \\
\hline$p_{43}$ & .20 & .23 & 10.2 & 9.4 & .22 & 9.9 & 11.3 & .37 & 22.5 & .20 & 4.9 & 4.4 & .20 & 5.0 & 4.7 & .41 & 23.8 \\
\hline
\end{tabular}

times 100 
1) $N=10,000$

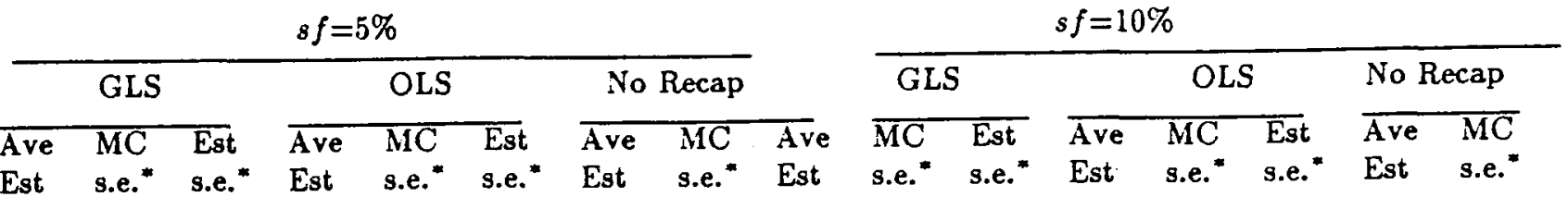

\begin{tabular}{|c|c|c|c|c|c|c|c|c|c|c|c|c|c|c|c|c|}
\hline .80 & .80 & 1.4 & 1.6 & .80 & 1.8 & 1.9 & .77 & 4.3 & .80 & .95 & .88 & .80 & 1.1 & 1.1 & .78 & 4.2 \\
\hline .10 & .11 & 1.6 & 1.5 & .10 & 1.8 & 1.9 & .14 & 2.7 & .10 & .84 & .84 & .10 & 1.0 & 1.0 & .14 & 2.5 \\
\hline .05 & .05 & 1.5 & 1.5 & .05 & 1.9 & 1.9 & .05 & 3.8 & .05 & .70 & .81 & .05 & .95 & 1.1 & .05 & 3.7 \\
\hline .05 & .06 & 4.7 & 4.8 & .06 & 5.1 & 5.7 & .20 & 22.8 & .05 & 1.9 & 2.3 & .05 & 2.1 & 2.4 & .17 & 21.3 \\
\hline .50 & .47 & 8.6 & 7.8 & .48 & 10.1 & 10.3 & .13 & 17.1 & .49 & 4.2 & 3.9 & .50 & 4.7 & 4.9 & .14 & 16.6 \\
\hline .40 & .42 & 8.8 & 8.0 & .40 & 9.5 & 10.3 & .46 & 32.0 & .41 & 4.3 & 4.0 & .40 & 4.9 & 4.9 & .52 & 29.9 \\
\hline .05 & .06 & 3.5 & 3.5 & .06 & 4.1 & 4.5 & .04 & 7.4 & .05 & 1.8 & 1.7 & .05 & 2.0 & 1.9 & .06 & 10.0 \\
\hline .05 & .05 & 3.3 & 3.6 & .05 & 3.5 & 4.2 & .18 & 14.2 & .05 & 1.8 & 1.8 & .05 & 2.0 & 1.9 & .22 & 15.1 \\
\hline .50 & .48 & 6.8 & 6.9 & .48 & 7.1 & 7.6 & .32 & 29.1 & .50 & 3.4 & 3.4 & .50 & 3.5 & 3.6 & .25 & 26.4 \\
\hline .05 & .05 & 3.4 & 3.0 & .06 & 3.8 & 3.9 & .04 & 5.3 & .05 & 1.5 & 1.6 & .05 & 1.7 & 1.8 & .03 & 5.1 \\
\hline .05 & .05 & 3.0 & 3.1 & .05 & 3.3 & 3.6 & .08 & 10.8 & .05 & 1.6 & 1.6 & .05 & 1.7 & 1.8 & .05 & 7.1 \\
\hline .20 & .21 & 4.9 & 5.5 & .21 & 5.1 & 6.0 & .32 & 21.7 & .20 & 3.2 & 2.7 & .20 & 3.2 & 2.9 & .36 & 20.1 \\
\hline
\end{tabular}




\section{Example Application}

\subsection{Background and Data}

The data shown in Tables 3.1 and 3.2 were collected as part of a CDCsponsored study of the potential benefits of an intervention program attempting to modify sex- and drug-related behavior toward preventing the spread of the AIDS virus. Behavior was measured in terms of a composite index of several attitude/action criteria. (see $D i$ Clemente et al (1985)). This index is here termed "stage", and has four possible values (the higher the stage, the better the behavior):

Stage $=1$, means pre-contemplative (i.e. not thinking about changing behavior)

$=2$, means contemplating making a change in behavior

$=3$, means ready to make a change in behavior

$=4$, means maintaining change in behavior.

This component of the study, which targeted self-identified intravenous drug users, was conducted in two geographically disjoint study areas, each consisting of several square city blocks in Long Beach, California. One area, designated the "Intervention" area, was subjected to a variety of intervention efforts, ranging from billboards, pamphlets and posters to visits by social workers conducting various educational discussions. The other area, designated as the "Comparison area", underwent no studysponsored intervention. The comparision area served as a control, to allow an assessment of the benefits of the intervention, over and above the "background" AIDS - related information at large in (and hopefully consistent across) both study areas.

The study was conducted over a period of about two years, and consisted of repeated surveys (or waves) in each study area at roughly six-week intervals. Each survey wave was conducted by one or more interviewers standing on street corners. The survey design called for interviewing every fifth person who was willing to be interviewed. Since the survey was rather long and necessarily dealt with sensitive personal information, a $\$ 5.00$ payment was made to respondents as an incentive to participate. Efforts were made to prevent people from participating more than once in each survey wave. However, although they were not intentionally sought 
out, people occasionally were selected for interview in consecutive waves, possibly due to the monetary reward for participating. These haphazard recaptures provide the transition data shown in Tables 3.1 (b) and 3.2 (b), which was used to augment the aggregate data shown in part (a) of these tables.

Tables 3.1 and 3.2 go here

\subsection{Analysis Results}

Table 3.3 displays the transition probability estimates based on the gls estimate of $\underline{\beta}$ (recall Corollary 2.5) based on $r=8$ waves and $m=4$ response categories. Standard recapture estimates of the sampling fraction were about $20 \%$, so the estimated standard errors were deemed trustworthy in view of our Monte Carlo results. The lack of fit statistics (each on $21 \mathrm{df}$ ) were 30.2 and 35.7 , respectively, for the intervention and comparison areas, suggesting that the Markov model fit reasonably well.

Table 3.3. Transition Probability Estimates from

Long Beach AIDS - Prevention Study

\begin{tabular}{llll|ll} 
& & \multicolumn{2}{c}{ Intervention Area } & \multicolumn{2}{c}{ Comparison Area } \\
\cline { 3 - 6 }$i$ & $j$ & $\hat{p}_{i j}^{(G L S)}$ & s.e. & $\widehat{p}_{i j}^{(G L S)}$ & s.e. \\
\hline 1 & 1 & .23 & .10 & .46 & .06 \\
1 & 2 & .06 & .07 & .08 & .04 \\
1 & 3 & .65 & .11 & .42 & .07 \\
1 & 4 & .07 & .08 & .04 & .02 \\
2 & 1 & .01 & .02 & .27 & .13 \\
2 & 2 & .54 & .12 & .28 & .11 \\
2 & 3 & .20 & .15 & .29 & .13 \\
2 & 4 & .25 & .13 & .16 & .08 \\
3 & 1 & .10 & .03 & .17 & .04 \\
3 & 2 & .02 & .02 & .06 & .03 \\
3 & 3 & .70 & .04 & .76 & .05 \\
3 & 4 & .18 & .03 & .00 & .04 \\
4 & 1 & .05 & .04 & .00 & .01 \\
4 & 2 & .03 & .04 & .02 & .08 \\
4 & 3 & .37 & .07 & .52 & .17 \\
4 & 4 & .55 & .06 & .45 & .16
\end{tabular}

Looking at Table 3.3, one sees that the standard errors are still unacceptably large for some $i, j$. However, for evaluating the efficacy of 
the intervention, the transition matrix $\underline{P}=\left(p_{i j}\right)$, which measures the onestep (here one-wave) transition behavior, is not of primary interest. Rather, the eventual (or stationary) distribution is of more use. Standard Markov chain theory says that if such a stationary distribution exists, then it may be obtained as $\lim _{k \rightarrow \infty} \underline{P}^{k}$. We found that for our estimated matrices in Table 3.3 the powers stabilized by $k=8$, so we took the rows of $\underline{P}^{8}$ as the eventual distribution. Thus, we obtained estimates of the eventual distribution for each study area, with standard errors obtained by the delta method from the covariance matrices of the estimates in Table 3.3. These are displayed in Table 3.4, along with the initial distribution estimates from Tables $3.1(a)$ and $3.2(a)$.

Table 3.4. Initial and Eventual Distribution Estimates for Long Beach AIDS - Prevention Study (s.e's in paren)

\begin{tabular}{l} 
Intervention Area \\
\multicolumn{1}{l|}{} \\
\cline { 2 - 5 }
\end{tabular}

Note that due to the contractive nature of the map $\underset{\sim}{P} \rightarrow \stackrel{\sim}{P}^{8}$ for stochastic matrices $P$, the standard errors of the eventual estimates are acceptably small. Also recall that by Markov theory, the eventual distribution is independent of the initial distribution, so that although the initial distributions differ substantially, the eventual distributions are nonetheless comparable for assessment of the impact of the intervention if it were to be maintained indefinitely. A standard Wald test of the equality of these eventual distributions gives a $\chi_{3}^{2}$ statistic of 67.6 
$(p=0)$, and examination of these distributions indicates that the intervention area eventually will have about $24 \%$ more of the population in stage 4 than will the comparison area.

ACKNOVLEDGEMENT: The authors thank Martin A. krepcho, Ph.D., Anne C. Freeman, M.S.P.H. and Elvin Magee, M.S. of the Dallas County Health Department for suggesting this research. They also thank the Center for Disease Control for permission to use the data of section 3 .

\section{References}

Apostol, T.M. (1974). Mathematical Analysis.

Bishop, Y.M.M., Fienberg, S.E. and Holland, P.W. (1975).Discrete Multivariate Analysis : Theory and Practice MIT Press.

Cochran, W.G. (1977). Sampling Techniques. Wiley.

Di Clemente, C.C., Prochaska, J.0. and Gibertini, M. (1985). Self-efficacy and the stages of self-change of smoking. Cognitive Therapy and Research 9 , 181-200.

Hajek, J. (1960). Limiting distributions in simple random sampling from a finite population. Publications of the Mathematics Institute of the Hungarian Academy of Science 5, 361-374.

Hajek, J. (1962). Asymptotically most powerful rank tests. Ann. Math. Stat.33, 1124-1147.

Hawkins, D.L., Han, C.P. and Eisenfeld, J. (1995). Estimating transition probabilities from aggregate samples augmented by haphazard matches. Technical report \# 302. Department of Mathematics, University of TexasArlington, Arlington, TX 76019.

Hajek, J. (1968). Asymptotic normality of simple linear rank statistics under alternatives.Ann. Math. Stat. 39,325-46.

Jennrich, R.I. (1969). Asymptotic properties of nonlinear least squares estimators. Ann. Math. Stat. 40,633-643.

Kabfleish, J.D. and Lawless, J.F. (1984). Least squares estimation of transition probabilities from aggregate data. The Canadian Journal of Statistics 12, 169-182.

Lawless, J.F. and McLeish, D.L. (1984). The information in aggregate data from Markov chains. Biometrika 71, 419-430. 
Lee, T.C., Judge, G.G. and Zellner, A. (1977). Estimating the parameters of the Markov probability model from aggregate time series data. North Holl land.

Madow, W.G. (1948). On the limiting distributions of estimates based on samples from finite universes. Annals of Mathematical Statistics 19, 535545 .

Rosen, B. (1965). Limit theorems for sampling from finite populations. Arkiv for Matematik 5, 383-424.

Serfling, R.J. (1980). Approximation Theorems of Mathematical Statistics Wiley.

White, D.B. (1988). An application of a multivariate central limit theorem to sampling without replacement. Journal of Multivariate Analysis 24, 123128 . 
Table 3.1 Data For Intervention Area

(a) Aggregate Data

Stage $\quad$ Marginal fraction $\widehat{q}_{t i}$

\begin{tabular}{r|l|llll|llll}
\hline wave & $n_{t}$ & 1 & 2 & 3 & 4 & $i=1$ & 2 & 3 & 4 \\
\hline $\mathrm{t}=1$ & 145 & 20 & 20 & 61 & 44 & .14 & .14 & .42 & .30 \\
2 & 114 & 16 & 15 & 50 & 33 & .14 & .13 & .44 & .29 \\
3 & 123 & 16 & 17 & 59 & 31 & .13 & .14 & .48 & .25 \\
4 & 106 & 8 & 7 & 43 & 48 & .07 & .07 & .41 & .45 \\
5 & 103 & 13 & 7 & 57 & 26 & .13 & .07 & .55 & .25 \\
6 & 104 & 17 & 7 & 55 & 25 & .16 & .07 & .53 & .24 \\
7 & 112 & 3 & 3 & 80 & 26 & .03 & .03 & .71 & .23 \\
8 & 120 & 14 & 6 & 69 & 31 & .12 & .04 & .58 & .26
\end{tabular}

(b) Transition Data from Haphazard Recaptures

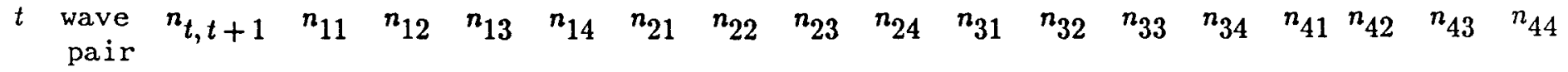

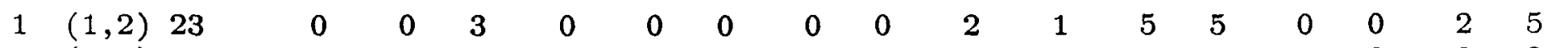

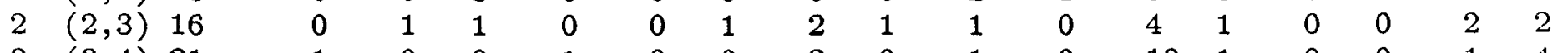

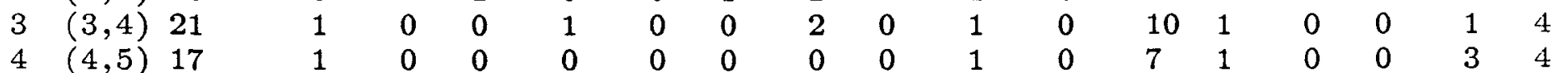

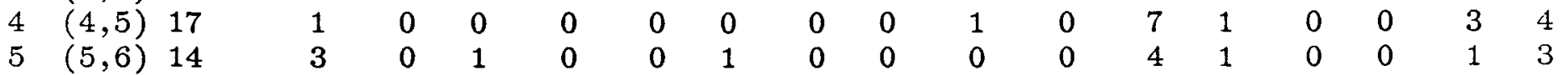

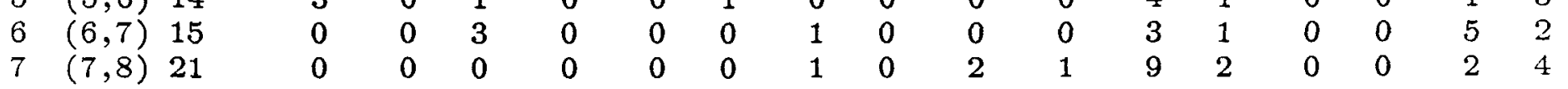

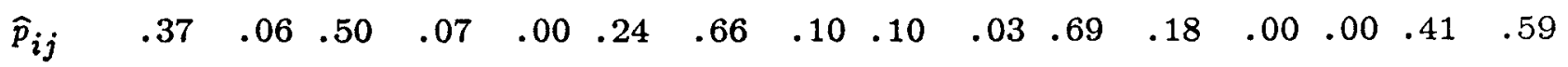


Table 3.2. Data for Comparison Area

(a) Aggregate Data

Stage Marginal fraction $\widehat{q}_{t i}$

\begin{tabular}{c|l|llll|llll}
\hline wave & $n_{t}$ & 1 & 2 & 3 & 4 & $i=1$ & 2 & 3 & 4 \\
\hline$t=1$ & 148 & 47 & 15 & 63 & 23 & .32 & .10 & .43 & .15 \\
2 & 103 & 16 & 12 & 62 & 13 & .16 & .12 & .60 & .12 \\
3 & 131 & 31 & 14 & 65 & 21 & .24 & .11 & .50 & .16 \\
4 & 118 & 25 & 18 & 59 & 16 & .21 & .15 & .50 & .14 \\
5 & 114 & 31 & 13 & 55 & 15 & .27 & .11 & .48 & .13 \\
6 & 127 & 29 & 16 & 72 & 10 & .23 & .13 & .57 & .08 \\
7 & 152 & 37 & 17 & 77 & 21 & .24 & .11 & .51 & .14 \\
8 & 147 & 25 & 5 & 110 & 7 & .17 & .03 & .75 & .05
\end{tabular}

(b) Transition Data from Haphazard Recaptures

$t$ wave $\begin{array}{llllllllllllllllll}\text { pair } & n_{t, t+1} & n_{11} & n_{12} & n_{13} & n_{14} & n_{21} & n_{22} & n_{23} & n_{24} & n_{31} & n_{32} & n_{33} & n_{34} & n_{41} & n_{42} & n_{43} & n_{44}\end{array}$

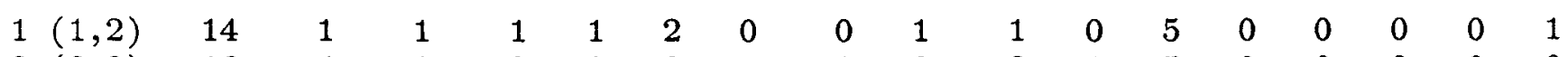

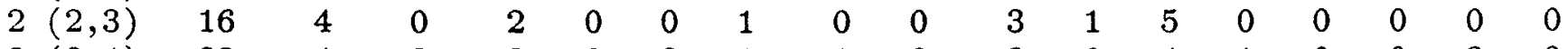

$\begin{array}{llllllllllllllllll}3(3,4) & 22 & 4 & 2 & 3 & 0 & 2 & 1 & 1 & 0 & 2 & 0 & 4 & 1 & 0 & 0 & 2 & 0\end{array}$

$\begin{array}{llllllllllllllllll}4(4,5) & 23 & 5 & 0 & 2 & 0 & 0 & 0 & 2 & 0 & 4 & 0 & 8 & 1 & 0 & 0 & 1 & 0\end{array}$

$\begin{array}{llllllllllllllllll}5(5,6) & 20 & 6 & 0 & 4 & 0 & 1 & 0 & 0 & 0 & 2 & 1 & 4 & 0 & 0 & 0 & 1 & 1\end{array}$

$\begin{array}{llllllllllllllllll}6(6,7) & 21 & 2 & 1 & 1 & 0 & 0 & 1 & 1 & 0 & 2 & 2 & 7 & 2 & 0 & 0 & 1 & 1 \\ 7(7,8) & 17 & 9 & 1 & 1 & 0 & 1 & 1 & 0 & 0 & 2 & 1 & 1 & 0 & 0 & 0 & 0 & 0\end{array}$

$\begin{array}{llllllllllllllllll}\widehat{p}_{i j} & .60 & .09 & .29 & .02 & .40 & .25 & .28 & .07 & .27 & .08 & .59 & .06 & .00 & .00 & .64 & .36\end{array}$ 


\section{Appendix}

We sketch here the proofs of the main theorems. Complete details are given in the $c$ technical report HHE.

Proof of Theorem 2.1

Since $\underline{Y}_{2 N}$ is a vector of ratios (see (2.9)), consider the expanded vector

$\underline{W}_{N}^{*}=\left[\underline{\underline{q}}_{N}^{* T}, \underline{R}_{N 1}^{T}, \cdots, \underline{R}_{N m}^{T}, D_{N 1}, \cdots, D_{N m}\right]^{T}$

where $\underline{R}_{N i}$ and $D_{N i}$ are defined in section 2.5.2. The asymptotic normality of $\underline{W}_{N}^{*}$ implies $\underline{W}_{N}$ by the delta method, so we show the former.

Lemma A.1. Under model (2.4) and assumptions (A1)-(A4),

$\underline{B}_{N}^{*}\left\{\underline{W}_{N}^{*}-E \underline{W}_{N}^{*}\right\} \stackrel{\mathcal{L}}{\rightarrow} N\left(\underline{0}, \underline{\Gamma}_{\underline{\omega}} *\right)$ as $N \rightarrow \infty$,

where $\underline{B}_{N}^{*}=:$ Block diag $\{n_{1}^{1 / 2} \underline{I}_{m-1}, \cdots, n_{r}^{1 / 2} \underline{I}_{m-1}, \underbrace{g_{N}^{1 / 2} \underline{I}_{m-1}, \cdots, g_{N}^{1 / 2} \underline{I}_{m-1}}_{m \text { times }}$,

$\left.g_{N}^{1 / 2} \underline{I}_{m}\right\}, g_{N}=: \sum_{t=1}^{r-1} n_{t} n_{t+1} / N$ and $\underline{\Gamma}_{\omega^{*}}$ is some positive definite covariance

matrix of no direct interest.

Proof of Lemma A.1. The idea is to show that any linear compound $\underline{\lambda}^{T} \underline{W}_{N}^{*}$ is asympt univariate normal. In this direction, recall that $\underline{\widehat{q}}_{N}^{*}=\left[\underline{\widehat{q}}_{1 N}, \cdots, \underline{\hat{q}}_{r N}^{T}\right]^{T}$, with $\underline{\widehat{q}}_{t N}$ given by 
and so is a vector of linear statistics in the random sample indicators $I_{l t}$. Asymptotic n of such statistics under our assumptions (A3) are well known; see White (1988), Hajek (1960 (1965) or Madow (1948). However, observe that

$\underline{R}_{N i}=\sum_{t=1}^{r-1} g_{N}{ }_{t}^{1} \sum_{l=1}^{N} z_{l t i} \underline{Z}_{l, t+1} I_{l t} I_{l, t+1} \quad$ and $D_{N i}=\sum_{t=1}^{r-1} g_{N}{ }_{t}^{1} \sum_{l=1}^{N} Z_{l t i} I_{l t} I_{l, t+1}$

are nonlinear statistics in the indicators $I_{l t}$, which apparently require a modified appr limit distribution theory. Fortunately, the method of Hajek $(1962,1968)$ for approximat statistics by sums of independent random variables can be extended to these statistics a the required result.

Hajek's method begins with the observation that statistics in the sampling indicators the same distributions as certain rank statistics. Specifically, for each $1 \leq t \leq r$ defin $U(0,1)$ variables $V_{N l}^{(t)}, l=1, \cdots, N$ and let $\underline{R}_{N}^{(t)}=:\left(R_{N 1}^{(t)}, \cdots, R_{N N}^{(t)}\right)$ denote the vector of ranks of

$V_{N 1}^{(t)}, \cdots, V N_{N}^{(t)}$. Then the random vector

$\underline{I}_{N t}^{*}=:\left(I\left(R_{N 1}^{(t)} \leq n_{t}\right), \cdots, I\left(R_{N N}^{(t)} \leq n_{t}\right)\right)$ has the same joint distribution as does

$\underline{I}_{N t}=:\left(I_{1 t}, \cdots, I_{N t}\right)$ (conditional on $\left.\sum_{l=1}^{N} I_{l t}=n_{t}\right)$. Further, if (as we

assume $\underline{I}_{N 1}, \cdots, \underline{I}_{N r}$ are independent, and if $\{V(t)\}$ are independent across $t=1, \cdots, r$, then the $\underline{I}_{N}^{*}=:\left(\underline{I}_{N 1}^{*}, \cdots, \underline{I}_{N r}^{*}\right)$ and $\underline{I}_{N}=:\left(\underline{I}_{N 1}, \cdots, \underline{I}_{N r}\right)$ have the same distribution, as do $h\left(\underline{I}_{N}^{*}\right)$ and $h\left(\underline{I}_{N}\right)$ measurable map $h$. The implication of this here is that for the limit distribution of $W_{N}^{*}$, assume that the component statistics are defined in terms of $\underline{I}_{N}^{*}$ rather than $\underline{I}_{N}$ ( as currently given). 
The next step in Hajek's method is to approximate the resulting rank statistics in independent random variables. For linear statistics like the $\underline{\widehat{q}}_{t}$ (with $\underline{I}_{N t}^{*} \operatorname{replacing} \underline{I}_{N}$ the above discussion), we have Hajek's basic lemma, given here as reformulated by White (1 adapted to the present context.

$\underline{\text { Lemma }} \underline{A .2}$. For each $t \epsilon\{1, \cdots, r\}$ and any scalar array $\left\{X_{N k t}: 1 \leq k \leq N\right\}$ satisfying $($ as $N \rightarrow \infty)$

(i) $n_{t}^{1 / 2} \bar{X}_{N t \rightarrow 0,} \bar{X}_{N t}=: \frac{1}{N} \sum_{k=1}^{N} X_{N k t} ;$ and

(ii) $n_{t}^{1 / 2} S_{N t \rightarrow 0,}^{2} S_{N t}^{2}=: \frac{1}{N-1} \sum_{k=1}^{N}\left(X_{N k t}-\bar{X}_{N t}\right)^{2}$,

we have $E\left(U_{N t}-U_{N t}^{*}\right)^{2} \rightarrow 0$, where

$U_{N t}=\sum_{k=1}^{N} X_{N k t} I\left(R_{N k}^{(t)} \leq n_{t}\right), \quad U_{N t}^{*}=\sum_{k=1}^{N} X_{N k t} I\left(V_{N k}^{(t)} \leq n_{t} / N\right)$

Remark. Lemma A.2. reduces the limit distribution problem for linear statistics $U_{N t}$ to $U_{N t}^{*}$, which is a linear combination of the independent random variables $\beta_{N k t}^{*}=: I\left(V_{N k}^{(t)} \leq n_{t} / N\right)$, is amenable to the central limit theorem for triangular arrays. The following result, $p$ HHE, extends Lemma A.2 to statistics of the form $\underline{R}_{N i}$ and $D_{N i}$. Define $\beta_{N k t}=: I\left(R_{N k}^{(t)} \leq n_{t}\right)$.

Lemma A.3. If the arrays $\left\{X_{N k t}: 1 \leq k \leq N\right\}, t=1, \cdots, r$ each satisfy conditions (i) and (ii) of Le and if, for $1 \leq t \leq r-1$,

$\xi_{N t}=\sum_{k=1}^{N} X_{N k t} \beta_{N k t} \beta_{N k, t+1}$, 
$\xi_{N t}^{*}=\sum_{k=1}^{N} X_{N k t} \beta_{N k t}^{*} \beta_{N k, t+1}^{*}$

then as $N \rightarrow \infty$,

(a) $E\left(\xi_{N t}-\xi_{N t}^{*}\right)^{2} \rightarrow 0, \quad 1 \leq t \leq r-1$; and

(b) $E\left\{\sum_{t=1}^{r-1} \xi_{N t}-\sum_{t=1}^{r-1} \xi_{N t}^{*}\right\}^{2} \rightarrow 0$.

To apply these results to $\underline{W}_{N}^{*}$ (with ranks substituted), we first need to decomp component statistics appropriately. We have, for $1 \leq t \leq r$,

$\widehat{\hat{\alpha}}_{N t}=: n_{t}^{1 / 2}\left\{\hat{\underline{q}}_{N t}-E \widehat{q}_{N t}\right\}=n_{t}-1 / 2 \sum_{l=1}^{N}\left(\underline{Z}_{l t}-\underline{\bar{Z}}_{t}^{(N)}\right) \beta_{N l t}=: \sum_{l=1}^{N} \underline{1}_{N l t} \underline{X}_{N l t}$, where

${ }_{1} \underline{X}_{N l t}=n_{t}^{-1 / 2}\left(\underline{Z}_{l t}-\underline{\bar{Z}}_{t}^{(N)}\right)$ and $\underline{\bar{Z}}_{t}^{(N)}=: \frac{1}{N} \sum_{l=1}^{N} \underline{Z}_{l t}$. Further, for $1 \leq i \leq m$,

$\widehat{\widehat{T}}_{N i}=: g_{N}^{1 / 2}\left\{\underline{R}_{N i}-E \underline{R}_{N i}\right\}$

$$
\begin{aligned}
& =g_{N}^{1 / 2}\left\{\sum_{t=1}^{r-1} g_{N}^{-1} \sum_{l=1}^{n} \underline{c}_{l t i} \beta_{N l t} \beta_{N l, t+1}-\sum_{t=1}^{r-1} \underline{\bar{c}}_{i t}^{(N)}\right\}
\end{aligned}
$$

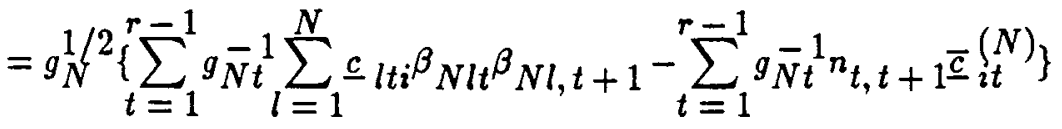

$$
\begin{aligned}
& +g_{N}^{1 / 2}\left\{\sum_{t=1}^{r-1} g_{N} \overline{1}^{1} n_{t, t+1} \overline{\underline{c}}_{i t}^{(N)}-\sum_{t=1}^{r-1} \overline{\bar{c}} i t\right\} \\
& =\sum_{t=1}^{r} \sum_{l=1}^{N} g_{N}^{1 / 2} g_{N t}{ }^{1}\left\{\underline{c}_{i l t}-\underline{\bar{c}}_{i t}^{(N)}\right\} \beta_{N l t}{ }^{\beta} N l, t+1
\end{aligned}
$$




$$
\begin{aligned}
& +g_{N}^{1 / 2} \sum_{t=1}^{r-1} \frac{\bar{c}_{i t}^{(N)}}{g_{N t}}\left\{\sum_{l=1}^{N} \beta_{N l t} \beta_{N l, t+1}-n_{t} n_{t+1} / N\right\} \\
& =: \sum_{t=1}^{r-1} \sum_{l=1}^{N}{ }_{2} \underline{X}_{N l t}^{(i)}{ }^{\beta}{ }_{N l t}{ }^{\beta} N l, t+1 \quad\left(\operatorname{call} \text { this }{ }_{1} \widehat{\widehat{\tau}}_{N i}\right) \\
& \left.+\sum_{t=1}^{r-1}{ }_{2} \underline{d}_{N t i} \sum_{l=1}^{N}\left\{\beta_{N l t^{\beta}}{ }_{N l, t+1}-E\left(\beta_{N l t}{ }_{N l, t+1}\right)\right\} \text { (call this }{ }_{2} \hat{\underline{\tau}}_{N i}\right)
\end{aligned}
$$

where ${ }_{2} \underline{X}_{N l t}^{(i)}=: g_{N}^{1}{ }^{2} g_{N}{ }_{t}^{1}\left\{\underline{c}_{i l t}-\underline{\bar{c}}_{i t}^{(N)}\right\}, \underline{\bar{c}}_{i t}^{(N)}=: \frac{1}{N} \sum_{l=1}^{N} \underline{\underline{c}}_{i l t}$

and ${ }_{2} \underline{\underline{d}}_{N t i}=g_{N}^{1 / \underline{\underline{c}}_{i t}}(N) / g_{N t}$. Similarly, for $1 \leq i \leq m$,

$$
\begin{aligned}
& \widehat{\delta}_{N i}=: g_{N}^{1 / 2}\left\{D_{N i}-E D_{N i}\right\} \\
& \left.=\sum_{t=1}^{r-1} \sum_{l=1}^{N}{ }_{3} \underline{X}_{N l t}^{(i)}{ }^{\beta} N l t^{\beta} N l, t+1 \text { (call this }{ }_{1} \widehat{\delta}_{N i}\right) \\
& \left.+\sum_{t=1}^{r-1}{ }_{3}^{d}{ }_{N t i} \sum_{l=1}^{N}\left\{\beta_{N l t}{ }^{\beta} N l, t+1-E\left(\beta_{N l t}{ }_{N l, t+1}\right)\right\} \quad \text { (call this }{ }_{2} \widehat{\delta}_{N i}\right)
\end{aligned}
$$$$
\text { where }{ }_{3} X_{N l t}^{(i)}=: g_{N}^{1 / 2} g_{N t}{ }_{1}\left\{Z_{l t i}-\bar{Z}_{i t}^{(N)}\right\}, \bar{Z}{ }_{i t}^{(N)}=: \frac{1}{N} \sum_{l=1}^{N} Z_{i l t}
$$

and ${ }_{3}^{d} N t i=: g_{N}^{1 / 2} \bar{Z}_{i t}^{(N)} / g_{N t}$.

The next step is to obtain approximations to $\underline{\underline{\alpha}}_{N t}, \widehat{\tau}_{N i}$ and $\widehat{\delta}_{N i}$ in terms of the ind variables $\beta_{N l t}^{*}$. In this direction, we have first that

$E\left\|\underline{\underline{\alpha}}_{N t}-\underline{\underline{\alpha}}_{N t}^{*}\right\|^{2} \rightarrow 0, \quad E\left\|_{1} \widehat{\widehat{\tau}}_{N i}-\widehat{\widehat{\tau}}_{N i}^{*}\right\|^{2} \rightarrow 0, \quad E\left\|_{1} \widehat{\delta}_{N i}-{ }_{1} \widehat{\delta}_{N i}^{*}\right\|^{2} \rightarrow 0$ 
by Lemma A.2, Lemma A.3 and Lemma A.3, respectively, where $\underline{\widehat{\alpha}}_{N t}^{*}{ }_{1} \widehat{\underline{I}}_{N i}^{*}$ and ${ }_{1} \widehat{\delta}_{N i}^{*}$ are the same corresponding "unstarred" quantities, but with $\beta_{N l t}$ replaced by $\beta_{N l t}^{*}$. Results (A.6) verifying that each of the arrays

${ }_{1} \underline{X}_{N l t}{ }_{2} \underline{X}_{N l t}$ and ${ }_{3} X_{N l t}$ satisfies conditions $(i)$ and (ii) of Lemma A.2. This is done in HHE.

The approximation is completed if we can find suitable approximations for ${ }_{2} \widehat{\underline{I}}_{N i}$ in (A.3) in (A.5). Note that Lemma A.3 does not apply to these quantities. Nevertheless, the $f$ result holds. (Proof in HHE)

$\underline{\text { Lemma }} \underline{\text { A.4. Let }}$

$$
\begin{aligned}
{ }_{2} \widehat{\widetilde{I}}_{N i}^{*} & =: \sum_{t=1}^{r-1}{ }^{2} \underline{\underline{d}}_{N t i} \sum_{l=1}^{N}\left\{\beta_{N l t}^{*} \beta_{N l, t+1}^{*}-E\left(\beta_{N l t}^{*} \beta_{N l, t+1}^{*}\right)\right\}, \\
{ }_{2} \widehat{\delta}_{N i}^{*}= & : \sum_{t=1}^{r-1}{ }^{d}{ }_{N t i} \sum_{l=1}^{N}\left\{\beta_{N l t}^{*} \beta_{N l, t+1}^{*}-E\left(\beta_{N l t}^{*} \beta_{N l, t+1}^{*}\right)\right\} .
\end{aligned}
$$

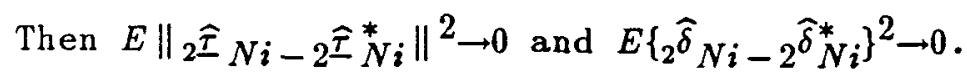

Results (A.6) and (A.7) imply that, for arbitrary $\underline{\lambda}$,

$E\left\{\underline{\lambda}^{T} \bullet \underline{B}_{N}^{*}\left[\underline{W}_{N}^{*}-E \underline{W}_{N}^{*}\right]-\underline{\lambda}^{T} \tilde{W}_{N}\right\}^{2} \rightarrow 0$, where

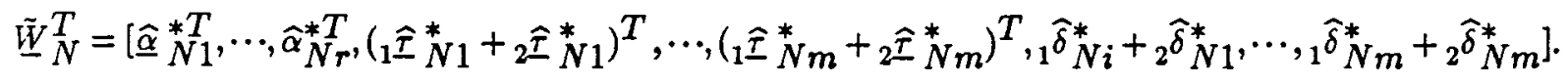

The asymptotic normality of $\underline{\lambda}^{T} \underline{\underline{W}}_{N}$ follows from the central limit theorem for triangular a independent random variables. (See the proof in HHE). Together with (A.8), this proves Lem 